\title{
Renormalization group studies of dense relativistic systems
}

\author{
Jens Braun, ${ }^{1,2,3}$ Timon Dörnfeld, ${ }^{1}$ Benedikt Schallmo, ${ }^{1,3}$ and Sebastian Töpfel $\oplus^{1,3}$ \\ ${ }^{1}$ Institut für Kernphysik, Technische Universität Darmstadt, D-64289 Darmstadt, Germany \\ ${ }^{2}$ ExtreMe Matter Institute EMMI, GSI, Planckstraße 1, D-64291 Darmstadt, Germany \\ ${ }^{3}$ Helmholtz Research Academy Hesse for FAIR, Campus Darmstadt, D-64289 Darmstadt, Germany
}

(Received 29 October 2020; accepted 20 September 2021; published 2 November 2021)

\begin{abstract}
Dense relativistic matter has attracted a lot of attention over many decades now, with a focus on an understanding of the phase structure and thermodynamics of dense strong-interaction matter. The analysis of dense strong-interaction matter is complicated by the fact that the system is expected to undergo a transition from a regime governed by spontaneous chiral symmetry breaking at low densities to a regime governed by the presence of a Cooper instability at intermediate and high densities. Renormalization group (RG) approaches have played and still play a prominent role in studies of dense matter, in general. In the present work, we study RG flows of dense relativistic systems in the presence of a Cooper instability and analyze the role of the Silver-Blaze property. In particular, we critically assess how to apply the derivative expansion to study dense-matter systems in a systematic fashion. This also involves a detailed discussion of regularization schemes. Guided by these formal developments, we introduce a new class of regulator functions for functional RG studies which is suitable to deal with the presence of a Cooper instability in relativistic theories. We close by demonstrating its application with the aid of a simple quark-diquark model.
\end{abstract}

DOI: $10.1103 /$ PhysRevD.104.096002

\section{INTRODUCTION}

A quantitative understanding of relativistic fermions in a dense environment is of great importance for many research fields, ranging from condensed-matter physics over nuclear physics to high-energy physics. With respect to stronginteraction matter, the potential existence of a color-superconducting ground state at supranuclear densities put forward in the 1970s has inspired uncounted studies at the interface of nuclear physics and astrophysics (see Ref. [1] for an early review). The interest in this state of matter even received a significant boost as a series of seminal works in the late 1990s [2-13] suggested the emergence of a rich plethora of symmetry-breaking patterns at high densities together with the formation of sizeable pairing gaps of $\sim 100 \mathrm{MeV}$ giving rise to large phase transition temperatures, see Refs. [14-22] for reviews. For the analysis of these symmetry-breaking patterns and the role of the Cooper instability associated with the formation of a pairing gap, renormalization group (RG) approaches have played and still play a very important role $[4,7,12,23,24]$, as they also do in condensed-

Published by the American Physical Society under the terms of the Creative Commons Attribution 4.0 International license. Further distribution of this work must maintain attribution to the author(s) and the published article's title, journal citation, and DOI. Funded by SCOAP . matter theory (see Refs. [25-28] for reviews). Moreover, RG studies provide us with detailed insights into the phase structure and thermodynamics of the theory of the strong interaction at lower densities (see, e.g., Refs. [23,24,29-44] for some recent advances and Ref. [45] for a review) as well as with constraints from quark-gluon dynamics for the equation of state of strong-interaction matter over a wide range of densities [46].

The central object for a field-theoretical description of quantum systems is the so-called quantum effective action. Its computation requires a suitable regularization and renormalization procedure. In general, the regularization prescription generates terms depending on a UV cutoff scale $\Lambda$ which are then absorbed as counter terms in the underlying bare action as part of the renormalization procedure. With respect to studies of systems at finite density, one may now be worried that the counter terms depend on the chemical potential. However, this is not necessarily the case. Indeed, it turns out that the partition function is invariant under a change of the chemical potential, provided the latter does not exceed a critical value $[34,47]$. This is known as the Silver-Blaze property of quantum field theories [48]. Assuming that the regularization prescription does not violate the symmetry associated with this invariance, it follows that the counter terms are indeed independent of the chemical potential $[34,47]$.

In RG studies, which are at the heart of the present work, fluctuations are integrated out successively around a given 
point in momentum space. This procedure implicitly defines a regularization and renormalization prescription. More specifically, we may choose to integrate out fluctuations from high- to low-momentum scales or around a suitably chosen finite scale, for example, the scale set by the chemical potential $\mu$ in case of studies of dense matter. Apparently, this choice is delicate since it may induce an explicit breaking of symmetries. For example, it may be a natural choice to integrate out fluctuations around the Fermi scale $\mu$ in the presence of a Cooper instability. By this, however, we break explicitly the symmetry associated with the aforementioned Silver-Blaze property of quantum field theories, as we discuss below.

These are still very general statements which do not yet take into account the fact that an actual computation of the quantum effective action usually involves approximations. A prominent and widely used approximation scheme is the so-called derivative expansion, basically corresponding to an expansion of correlation functions in terms of their external momenta. Such an expansion ultimately requires the specification of an expansion point. A priori, this point is at our disposal but, loosely speaking, should be chosen such that a low-order expansion already allows us to capture the most relevant dynamics of the system under consideration. Of course, a bias is introduced when we choose a specific expansion point and therefore this has to be considered with great care. Also, the chosen expansion point may violate symmetries as the one associated with the Silver-Blaze property.

The focus of the present work is on issues which may arise in RG studies of dense relativistic matter in the presence of a Cooper instability. To this end, we begin our discussion by reviewing and extending previous general studies [34,47,49] of the Silver-Blaze property and its consequences from a phenomenological as well as fieldtheoretical standpoint in Sec. II. In Sec. III, we then analyze how the Silver-Blaze property and the choice of a specific expansion point in the derivative expansion affects the theoretical predictions for systems where a Cooper instability is expected to govern the underlying dynamics. From this analysis, we deduce that, in the RG flow, fluctuations should be integrated out around the Fermi surface in order to recover the expected Bardeen-Cooper-Schrieffer (BCS) scaling behavior of observables, at least in studies based on a derivative expansion of the quantum effective action. Based on these findings, we then construct a new class of regulators for functional RG studies in Sec. IV. This class of regulators is suitable to tackle systems governed by the presence of a Cooper instability and allows us to recover the expected BCS scaling behavior. Its application is demonstrated with the aid of a simple quark-diquark model in Sec. V. Our conclusions including a discussion of the application of the derivative expansion for studies of the theory of the strong interaction, i.e., quantum chromodynamics (QCD), over a wide range of densities can be found in Sec. VI.

\section{SILVER-BLAZE PROPERTY}

Let us consider a system of fermions and (complex) scalar fields which both couple to a chemical potential $\mu$. This implies that the scalar fields also carry a finite "fermion number" $F$ (or baryon number $B$ in case of QCD), i.e., phenomenologically speaking, the scalars may be considered as composites of fermions. Examples for such fields are diquark fields which carry fermion number $|F|=2$ (or baryon number $|B|=2 / 3$ ). On the other hand, pions composed of a quark and an antiquark carry net fermion number $F=0$ (baryon number $B=0$ ). The sign of $F$ depends on whether the scalar fields are "composed" of two fermions or two antifermions. Of course, both the fermions and the scalar fields may also carry additional quantum numbers, for example, color in QCD. In any case, we now consider an action of the following form:

$$
\begin{aligned}
& S\left[\bar{\psi}, \psi, \phi^{*}, \phi\right] \\
& =\int_{x}\left\{\bar{\psi}\left(\mathrm{i} \gamma_{0}\left(\partial_{0}+\mu\right)+\mathrm{i} \gamma_{j} \partial_{j}\right) \psi\right. \\
& \left.\quad+\left[\left(\partial_{0}-F \mu\right) \phi^{*}\right]\left[\left(\partial_{0}+F \mu\right) \phi\right]+\left(\partial_{j} \phi^{*}\right)\left(\partial_{j} \phi\right)\right\} \\
& \quad+V_{\text {int }}\left[\bar{\psi}, \psi, \phi^{*}, \phi\right],
\end{aligned}
$$

where $\int_{x}=\int \mathrm{d}^{4} x$, and $V_{\text {int }}$ describes a set of interactions. Without loss of generality, we assume $\mu>0$. Moreover, we note that we employ Euclidean $\gamma$ matrices with $\gamma_{\mu}^{\dagger}=\gamma_{\mu}$.

The kinetic terms of the action $S$ are invariant under the following set of transformations of the fields and the chemical potential,

$$
\begin{aligned}
\bar{\psi} & \mapsto \bar{\psi} \mathrm{e}^{-\mathrm{i} \alpha x_{0}}, \\
\phi^{*} & \mapsto \phi^{*} \mathrm{e}^{-\mathrm{i} F \alpha x_{0}}, \quad \phi \mapsto \mathrm{e}^{\mathrm{i} \alpha x_{0}} \psi, \\
& \mapsto \mathrm{e}^{\mathrm{i} F \alpha x_{0}} \phi,
\end{aligned}
$$

and

$$
\mu \mapsto \mu-\mathrm{i} \alpha,
$$

where $\alpha \in \mathbb{R}$ is a constant "angle", and we have promoted $\mu$ to a complex quantity. We refer to Eqs. (2)-(4) as SilverBlaze transformations. Assuming that also the interaction terms contained in $V_{\text {int }}$ are invariant under this transformation, we conclude that the entire action $S$ is invariant.

Looking at the Silver-Blaze transformation and the action (1), one may be tempted to treat the chemical potential as a constant background (gauge) field and introduce corresponding "Silver-Blaze-covariant" derivatives of the form $D_{0}^{F}=\partial_{0}+F \mu$ (with $F \in \mathbb{Z}$ ). Unlike gauge fields, however, we do not integrate over the chemical potential in the path integral. The chemical potential is rather an external control parameter.

Let us begin our discussion with the zero-temperature limit and postpone the finite-temperature case to the end of 
this section. The dynamics of the quantum theory associated with the classical action $S$ is determined by the partition function. The latter is related to the following path integral:

$$
\mathcal{Z}=\mathcal{N} \int \mathcal{D} \bar{\psi} \mathcal{D} \psi \mathcal{D} \phi^{*} \mathcal{D} \phi \mathrm{e}^{-S+\int_{x} \mathcal{J}^{T} \cdot \varphi},
$$

where $\mathcal{N}$ is a normalization factor. The vector $\mathcal{J}^{T}=$ $\left(\bar{\eta},-\eta^{T}, J^{*}, J\right)$ contains the sources for the fermion and scalar fields, respectively, and $\varphi^{T}=\left(\psi^{T}, \bar{\psi}, \phi, \phi^{*}\right)$ is a collective field vector, only introduced to keep the notation in a compact form. As the action $S$ depends on the chemical potential, also the path integral $\mathcal{Z}$ depends on it. Evaluating the path integral $\mathcal{Z}$ for vanishing sources, we obtain the grand-canonical $(\mathrm{gc})$ partition function $\mathcal{Z}_{\mathrm{gc}}$.

We now assume that $\mathcal{Z}_{\mathrm{gc}}$ is analytic within some domain of values for the (potentially complex-valued) chemical potential. For concreteness, let us assume that $\mathcal{Z}_{\mathrm{gc}}$ is analytic for $\Re(\mu)<\mu_{\mathrm{c}}$. Below we see that the associated (real-valued) "critical" value $\mu_{\mathrm{c}}$ of the chemical potential is set by the (pole) mass and fermion number $F$ of those fields that couple to the chemical potential. From a phenomenological standpoint, this may not come unexpected at all. Indeed, the chemical potential is the change in free energy when fermions are added to or removed from the system. Considering, for example, a noninteracting system of fermions with mass $m_{\psi}$ for illustration, a lower bound for this change in the free energy is given by the mass of the fermions. We therefore expect that the fermion density (strictly speaking, the difference of the density of fermions and antifermions) can become finite only for $\mu \geq m_{\psi}$. For $\mu<m_{\psi}$, the density remains zero, and we expect the system to be invariant under a change of the chemical potential. In particular, this implies that the partition function $\mathcal{Z}_{\mathrm{gc}}$ does not depend on $\mu$ for $\mu<m_{\psi}$. This is known as the Silver-Blaze property [48].

Coming back to our general discussion, it follows from the invariance of the action $S$ and the measure of the path integral for the partition function $\mathcal{Z}_{\mathrm{gc}}$ under Silver-Blaze transformations that

$$
\left.\mathcal{Z}_{\mathrm{gc}}\right|_{\mu}=\left.\mathcal{Z}_{\mathrm{gc}}\right|_{\mu \rightarrow \mu-\mathrm{i} \alpha}
$$

i.e., the partition function is invariant under a shift of the chemical potential $\mu$ along the imaginary axis. Assuming that $\mathcal{Z}_{\mathrm{gc}}$ is analytic for $\Re(\mu-\mathrm{i} \alpha)<\mu_{\mathrm{c}}$, we can perform an analytic continuation of the parameter $\alpha, \alpha \rightarrow \mathrm{i} \alpha$ and conclude that $\mathcal{Z}_{\mathrm{gc}}$ does not depend on $\mu \in \mathbb{R}$ for $\mu<\mu_{\mathrm{c}}$. This may be viewed as the mathematical confirmation of our phenomenological line of arguments on the $\mu$ independence of $\mathcal{Z}_{\mathrm{gc}}$ given above.

Along the lines of Ref. [47], we can now also study the $\mu$ dependence of one-particle-irreducible (1PI) correlation functions. To this end, we recall that $\mathcal{Z}$ is a functional of the sources and a function of $\mu$. Using again the invariance of the action $S$ and the measure of the path integral under Silver-Blaze transformations, we find

$\left.\mathcal{Z}\left[\bar{\eta}, \eta, J^{*}, J\right]\right|_{\mu}=\left.\mathcal{Z}\left[\bar{\eta} \mathrm{e}^{-\mathrm{i} \alpha x_{0}}, \mathrm{e}^{\mathrm{i} \alpha x_{0}} \eta, J^{*} \mathrm{e}^{-\mathrm{i} F \alpha x_{0}}, \mathrm{e}^{\mathrm{i} F \alpha x_{0}} J\right]\right|_{\mu \rightarrow \mu-\mathrm{i} \alpha}$.

Considering now the Legendre transform of $\ln \mathcal{Z}$ with respect to the sources, we eventually arrive at the following relation for the quantum effective action $\Gamma$,

$$
\begin{aligned}
\Gamma\left[\bar{\Psi}_{\mathrm{cl}},\right. & \left.\Psi_{\mathrm{cl}}, \Phi_{\mathrm{cl}}^{*}, \Phi_{\mathrm{cl}}\right]\left.\right|_{\mu} \\
& =\left.\Gamma\left[\bar{\Psi}_{\mathrm{cl}} \mathrm{e}^{-\mathrm{i} \alpha x_{0}}, \mathrm{e}^{\mathrm{i} \alpha x_{0}} \Psi_{\mathrm{cl}}, \Phi_{\mathrm{cl}}^{*} \mathrm{e}^{-\mathrm{i} F \alpha x_{0}}, \mathrm{e}^{\mathrm{i} F \alpha x_{0}} \Phi_{\mathrm{cl}}\right]\right|_{\mu \rightarrow \mu-\mathrm{i} \alpha},
\end{aligned}
$$

where $\bar{\Psi}_{\mathrm{cl}}, \Psi_{\mathrm{cl}}, \Phi_{\mathrm{cl}}^{*}$, and $\Phi_{\mathrm{cl}}$ denote the so-called classical fields. These fields should not be confused with those which minimize the effective action. We can now take functional derivatives of Eq. (8) with respect to the classical fields to study the $\mu$ dependence of the $n$-point $1 \mathrm{PI}$ correlation functions.

To illustrate the consequences of Eq. (8) for correlation functions, let us take a functional derivative of Eq. (8) with respect to $\bar{\Psi}$ from the left and another one with respect to $\Psi$ from the right and evaluate the resulting expression on the ground state. Assuming that the latter is given by vanishing classical fields, we find for the fermionic two-point function in momentum space that

$$
\left.\Gamma_{\bar{\psi} \psi}^{(2)}\left(p_{0}, \vec{p}\right)\right|_{\mu}=\left.\Gamma_{\bar{\psi} \psi}^{(2)}\left(p_{0}-\alpha, \vec{p}\right)\right|_{\mu-\mathrm{i} \alpha} .
$$

Assuming further that $\Gamma_{\bar{\psi} \psi \psi}^{(2)}$ is analytic for $\Re(\mu-\mathrm{i} \alpha)<\mu_{\mathrm{c}}$, we can set $\alpha=-\mathrm{i} \mu$ and find

$$
\left.\Gamma_{\bar{\psi} \psi}^{(2)}\left(p_{0}, \vec{p}\right)\right|_{\mu}=\Gamma_{\bar{\psi} \psi}^{(2)}\left(p_{0}+\mathrm{i} \mu, \vec{p}\right) .
$$

For the two-point function associated with the complex scalar fields, we obtain

$$
\left.\Gamma_{\phi^{*} \phi}^{(2)}\left(p_{0}, \vec{p}\right)\right|_{\mu}=\Gamma_{\phi^{*} \phi}^{(2)}\left(p_{0}+\mathrm{i} F \mu, \vec{p}\right),
$$

provided that $\Gamma_{\phi^{*} \phi}^{(2)}$ is analytic for $\Re(\mu-\mathrm{i} F \alpha)<\mu_{\mathrm{c}}$. This analysis can be generalized to all $n$-point functions.

From this analysis, it follows that, at zero temperature and $\mu<\mu_{\mathrm{c}}$, the $\mu$ dependence of the correlation functions is trivially obtained by simply replacing the zeroth components of the four-momenta in the vacuum correlation functions with suitably $\mu$-shifted zeroth components, see, e.g., Eqs. (10) and (11). Because of the analytic properties of these functions, it also follows that they do not depend on $\mu$ for $\mu<\mu_{\mathrm{c}}$ at all. 
Let us now turn to the critical value $\mu_{\mathrm{c}}$ of the chemical potential. From our discussion of the two-point functions, we extract that the value of $\mu_{\mathrm{c}}$ is set by the pole mass and the fermion number $F$ of those fields which couple to the chemical potential. An analytic continuation of the theory in the complex $p_{0}$ plane is therefore restricted to the domain $\left|p_{0}\right|<\mu_{\mathrm{c}}$. Recall that the pole mass $m$ of a particle is defined by the position of the zero of its inverse propagator for $\vec{p}=0: \Gamma^{(2)}\left(p_{0}=\mathrm{i} m, 0\right)=0$. From our analysis, in particular, from Eqs. (10) and (11), we then deduce that $\mu_{\mathrm{c}}=\min \left\{m_{\psi}, m_{\phi} /|F|\right\}$. Here, $m_{\psi}$ and $m_{\phi}$ refer to the vacuum pole masses of the fermions and bosons, respectively.

Our findings have immediate consequences for the computation of the effective action. For example, with respect to the derivative expansion of the effective action, our analysis suggests that the associated expansion of the correlation functions in external momenta has to be performed around the point $\left(p_{0}+\mathrm{i} F \mu, \vec{p}\right)=(0,0)$ rather than $\left(p_{0}, \vec{p}\right)=(0,0)$ in order to preserve the Silver-Blaze property $[34,50]$. Thus, the expansion point is complex valued for $\mu>0$. An expansion around the point $\left(p_{0}, \vec{p}\right)=$ $(0,0)$ breaks explicitly the invariance under Silver-Blaze transformations. Our discussion implies that a Silver-Blazesymmetric derivative expansion requires to consider different expansion points in the complex $p_{0}$ plane in the underlying expansion of correlation functions. In Sec. III, we discuss issues related to the choice of the expansion point in detail.

Up to this point, we have ignored that a computation of the effective action requires a regularization and renormalization procedure. Of course, our conclusions following from the invariance of the theory under Silver-Blaze transformations only hold if this invariance is not violated within that procedure.

Let us now turn to RG studies of dense relativistic matter. The basic idea of RG studies is to integrate out fluctuations successively. For example, in the spirit of Wilson's idea of renormalization, we may integrate fluctuations from highto low-momentum scales. In principle, we may also integrate out fluctuations around the Fermi surface. The actual prescription specifying the details of the momentumshell integrations may lead to an explicit breaking of the invariance under Silver-Blaze transformations. In the following, we discuss this aspect by means of the Wetterich equation [51] which can be directly related to Wilson's approach to renormalization on the one-loop level.

The starting point for the derivation of the Wetterich equation [51] is obtained from Eq. (5) by inserting a regulator term $\Delta S_{k}$,

$$
\mathcal{Z}(k)=\mathcal{N} \int \mathcal{D} \bar{\psi} \mathcal{D} \psi \mathcal{D} \phi^{*} \mathcal{D} \phi \mathrm{e}^{-S-\Delta S_{k}+\int_{x} \mathcal{J}^{T} \cdot \varphi} .
$$

The new term $\Delta S_{k}$ is defined as

$\Delta S_{k}=\int_{x}\left\{\bar{\psi} R_{k}^{\psi}\left(\partial_{0}, \vec{\nabla}, \mu\right) \psi+\phi^{*} R_{k}^{\phi}\left(\partial_{0}, \vec{\nabla}, \mu\right) \phi\right\}$.

This term provides us with a suitable regularization of the path integral. In particular, it introduces the additional scale $k$ which screens infrared singularities. Anyhow, for $k \rightarrow 0$, we assume that the regulator functions appearing in $\Delta S_{k}$ vanish, i.e., $\lim _{k \rightarrow 0} R_{k}^{\psi / \phi}=0$, in order to ensure that the original path integral (5) is recovered in this limit. Note that the value of the path integral now depends on the scale $k$ and so do the correlation functions derived from it, including the partition function $\mathcal{Z}_{\mathrm{gc}}=\mathcal{Z}_{\mathrm{gc}}(k)$.

The regulator insertion (13) is bilinear in the fields. From this, we conclude that it does not break the invariance under Silver-Blaze transformations if the momentum-space representation of the regulator functions obeys

$$
R_{k}^{\psi}\left(\mathrm{i} p_{0}, \mathrm{i} \vec{p}, \mu\right)=R_{k}^{\psi}\left(\mathrm{i}\left(p_{0}-\mathrm{i} \mu\right), \mathrm{i} \vec{p}, 0\right),
$$

and

$$
R_{k}^{\phi}\left(\mathrm{i} p_{0}, \mathrm{i} \vec{p}, \mu\right)=R_{k}^{\phi}\left(\mathrm{i}\left(p_{0}-\mathrm{i} F \mu\right), \mathrm{i} \vec{p}, 0\right),
$$

where it is assumed that $R_{k}^{\psi}$ and $R_{k}^{\phi}$ are analytic functions of $p_{0}$. This is in line with the findings from our analysis of the $\mu$ dependence of the two-point functions.

Let us assume for a moment that the regulator functions obey these constraints and that their functional form is such that they introduce a mass gap $m_{\text {gap }} \sim k$ into the theory. By construction, this gap depends on the scale $k$ and screens divergences in the limit $p \rightarrow 0$ for $\mu=0$. Note that divergences of this type are screened by the chemical potential for $\mu>0$. In any case, we deduce that the regulator induces a shift of the pole positions of the propagators at finite $k$. It is important to add that the regulator should not lower the pole mass of the lowest lying state with a finite fermion number for $k>0$ since this potentially leads to a violation of the SilverBlaze property as well. Unfortunately, this appears to be a generic feature of conventionally used momentum cutoffs, see Ref. [34] for a discussion.

Since the fermion number $F$ together with the vacuum pole masses of those fields coupled to the chemical potential determine the critical value $\mu_{\mathrm{c}}$ of the chemical potential, the latter also becomes $k$ dependent, $\mu_{\mathrm{c}}=\mu_{\mathrm{c}}(k)$. For values of $k$ (much) greater than any of the pole masses, we even expect $\mu_{\mathrm{c}} \sim k$. For $k \rightarrow 0$, the $k$-dependent mass gaps in the propagator then approach the physical pole masses from above. For $k>0$, it follows by repeating our analysis for general correlation functions that the $k$-dependent correlation functions for finite $\mu$ are identical to those for $\mu=0$, provided that $\mu<\mu_{\mathrm{c}}(k)$. Indeed, for $\mu<\mu_{\mathrm{c}}(k)$, the $\mu$ dependence of the $k$-dependent correlation functions is obtained by simply replacing $p_{0}$ with $\left(p_{0}+\mathrm{i} F \mu\right)$ in the 
corresponding vacuum correlation functions. In particular, we find that $\mathcal{Z}_{\mathrm{gc}}(k)$ is constant for $\mu<\mu_{\mathrm{c}}(k)$. An important consequence of these observations regarding the $\mu$ dependence of the correlation functions is that the initial conditions for these functions at the scale $k=\Lambda>c_{\Lambda} \mu$ in the RG flow (i.e., the counter terms for the correlation functions in the terminology of renormalization theory) are identical to those in the limit $\mu \rightarrow 0$ [47]. The actual value of the constant $c_{\Lambda}>0$ depends on the details of the functional form of the regulator functions. In any case, even if the regulator preserves the Silver-Blaze symmetry, the expansion scheme employed to compute the effective action may still break the invariance under the SilverBlaze transformation. For example, as discussed above, a derivative expansion of the effective action around the point $\left(p_{0}, \vec{p}\right)=(0,0)$ breaks the invariance under such transformations.
In case of regulators which do not fulfill the constraints (14) and (15), the invariance under Silver-Blaze transformations is explicitly broken, and therefore, also the initial conditions of the RG flow, in general, depend on the chemical potential. While the dependence of the initial conditions on the chemical potential becomes parametrically suppressed if the initial scale is chosen sufficiently large, $\Lambda \gg \mu$, the explicit breaking of the Silver-Blaze symmetry by the regulator functions persists.

Let us now analyze the effect of Silver-Blaze transformations on the regularized path integral (12) for vanishing source terms; i.e., we consider the $k$-dependent partition function $\mathcal{Z}_{\mathrm{gc}}(k)$. Since the action $S$ and the measure of the corresponding path integral are invariant under SilverBlaze transformations, it suffices to study the variation of the regulator insertion $\Delta S_{k}$ under an infinitesimal SilverBlaze transformation. We find

$$
\begin{aligned}
& \Delta S_{k}\left[\bar{\psi}, \psi, \phi^{*}, \phi\right] \mapsto \Delta S_{k}\left[\bar{\psi}, \psi, \phi^{*}, \phi\right]+\alpha \int_{p} \bar{\Psi}(p)\left[\frac{\partial R_{k}^{\psi /}\left(\mathrm{i} p_{0}, \mathrm{i} \vec{p}, \mu\right)}{\partial p_{0}}-\mathrm{i} \frac{\partial R_{k}^{\psi}\left(\mathrm{i} p_{0}, \mathrm{i} \vec{p}, \mu\right)}{\partial \mu}\right] \Psi(p) \\
& \quad+\alpha \int_{p} \Phi^{*}(p)\left[F \frac{\partial R_{k}^{\phi}\left(\mathrm{i} p_{0}, \mathrm{i} \vec{p}, \mu\right)}{\partial p_{0}}-\mathrm{i} \frac{\partial R_{k}^{\phi}\left(\mathrm{i} p_{0}, \mathrm{i} \vec{p}, \mu\right)}{\partial \mu}\right] \Phi(p)+\mathcal{O}\left(\alpha^{2}\right),
\end{aligned}
$$

where $\int_{p}=\int \frac{\mathrm{d}^{4} p}{(2 \pi)^{4}}$. The Fourier transforms of the fields are defined by

$$
\psi(x)=\int_{q} \mathrm{e}^{\mathrm{i} q x} \Psi(q), \quad \bar{\psi}(x)=\int_{q} \mathrm{e}^{-\mathrm{i} q x} \bar{\Psi}(q),
$$

and

$$
\phi(x)=\int_{q} \mathrm{e}^{\mathrm{i} q x} \Phi(q), \quad \phi^{*}(x)=\int_{q} \mathrm{e}^{-\mathrm{i} q x} \Phi^{*}(q) .
$$

Note that $\Psi(q)=\Psi\left(q_{0}, \vec{q}\right)$ and $\Phi(q)=\Phi\left(q_{0}, \vec{q}\right)$. Moreover, we have assumed that the regulator functions $R_{k}^{\psi / \phi}$ can be expanded in terms of their arguments. Requiring

$$
\left.\left.\mathcal{Z}_{\mathrm{gc}}(k)\right|_{\mu} \stackrel{!}{=} \mathcal{Z}_{\mathrm{gc}}(k)\right|_{\mu \rightarrow \mu-\mathrm{i} \alpha}
$$

for $\Re(\mu-\mathrm{i} \alpha)<\mu_{\mathrm{c}}(k)$, we deduce from Eq. (16) that the 1PI two-point functions have to obey the following relation for $\Re(\mu-\mathrm{i} \alpha)<\mu_{\mathrm{c}}(k)$,

$$
\begin{aligned}
0= & \int_{p}\left(\frac{\delta^{2} \Gamma_{k}}{\delta \bar{\Psi}_{\mathrm{cl}} \delta \Psi_{\mathrm{cl}}}\right)_{0}^{-1}(p, p)\left[\frac{\partial R_{k}^{\psi}\left(\mathrm{i} p_{0}, \mathrm{i} \vec{p}, \mu\right)}{\partial p_{0}}-\mathrm{i} \frac{\partial R_{k}^{\psi}\left(\mathrm{i} p_{0}, \mathrm{i} \vec{p}, \mu\right)}{\partial \mu}\right] \\
& +\int_{p}\left(\frac{\delta^{2} \Gamma_{k}}{\delta \Phi_{\mathrm{cl}}^{*} \delta \Phi_{\mathrm{cl}}}\right)_{0}^{-1}(p, p)\left[F \frac{\partial R_{k}^{\phi}\left(\mathrm{i} p_{0}, \mathrm{i} \vec{p}, \mu\right)}{\partial p_{0}}-\mathrm{i} \frac{\partial R_{k}^{\phi}\left(\mathrm{i} p_{0}, \mathrm{i} \vec{p}, \mu\right)}{\partial \mu}\right]
\end{aligned}
$$

The subscript " 0 " indicates that the second functional derivatives of the effective action are evaluated on the minimum of the scale-dependent effective action $\Gamma_{k}$. For example, we have

$$
\left(\frac{\delta^{2} \Gamma_{k}}{\delta \Phi_{\mathrm{cl}}^{*} \delta \Phi_{\mathrm{cl}}}\right)_{0}^{-1}(p, q)=\left.\frac{\delta^{2} \ln \mathcal{Z}(k)}{\delta \tilde{J}^{*}(p) \delta \tilde{J}(q)}\right|_{\tilde{\mathcal{J}}=0}
$$


and similarly for the fermions. Here, $\tilde{J}$ and $\tilde{J}^{*}$ denote the Fourier transforms of the sources for the scalar fields, and correspondingly, $\tilde{\mathcal{J}}$ is the vector containing the Fourier transforms of all sources. For simplicity, we have assumed in Eq. (20) that the vacuum expectation values of the fields vanish in the minimum of the effective action $\Gamma_{k}$ for $\Re(\mu-\mathrm{i} \alpha)<\mu_{\mathrm{c}}(k)$. In any case, if the identity (20) is fulfilled in the regime defined by $\Re(\mu-\mathrm{i} \alpha)<\mu_{\mathrm{c}}(k)$, then $\mathcal{Z}_{\mathrm{gc}}(k)$ is constant and identical to its value at $\mu=0$ for $\mu<\mu_{\mathrm{c}}(k)$. The same holds for all $n$-point functions as discussed above.

We immediately deduce from Eq. (20) that the invariance under Silver-Blaze transformations is preserved if

$$
F \frac{\partial R_{k}^{\psi / \phi}\left(\mathrm{i} q_{0}, \mathrm{i} \vec{q}, \mu\right)}{\partial q_{0}}-\mathrm{i} \frac{\partial R_{k}^{\psi / \phi}\left(\mathrm{i} q_{0}, \mathrm{i} \vec{q}, \mu\right)}{\partial \mu}=0 .
$$

For example, this equation is fulfilled for the class of regulators implicitly defined by Eqs. (14) and (15).

Although Eq. (20) could potentially be used to derive constraints for the flow of the two-point functions to compensate for the regulator-induced violation of the Silver-Blaze symmetry in the spirit of Ward identities, we expect this equation to be only of limited use for concrete calculations. Indeed, an implementation of such constraints would require the knowledge of $\mu_{\mathrm{c}}(k)$ which is a dynamically determined quantity.

Finally, we would like to comment on the case of finite temperature. At finite temperature $T$ and chemical potential $\mu$, the continuous symmetry described by Eqs. (2)-(4) reduces to a discrete symmetry because of the compactification of the Euclidean time direction,

$$
\begin{aligned}
& \bar{\psi} \mapsto \bar{\psi} \mathrm{e}^{-2 \mathrm{i} n \pi T x_{0}}, \quad \psi \mapsto \mathrm{e}^{2 \mathrm{i} n \pi T x_{0}} \psi, \\
& \phi^{*} \mapsto \phi^{*} \mathrm{e}^{-2 \mathrm{i} F n \pi T x_{0}}, \quad \phi \mapsto \mathrm{e}^{2 \mathrm{i} F n \pi T x_{0}} \phi,
\end{aligned}
$$

and

$$
\mu \mapsto \mu+2 \mathrm{i} n \pi T,
$$

with $n \in \mathbb{Z}$. At finite temperature, we then have

$$
\left.\mathcal{Z}_{\mathrm{gc}}\right|_{\mu}=\left.\mathcal{Z}_{\mathrm{gc}}\right|_{\mu \rightarrow \mu+2 \mathrm{in} \pi T} .
$$

We add that we have $\mu \rightarrow \mu+(2 \mathrm{i} n \pi / N) T$ in $\mathrm{SU}(N)$ gauge theories [52]. For our analysis, it is now important to realize that the zeroth component of the Euclidean four-momentum is discrete at finite temperature. Hence, the analytic continuation that underlies our line of arguments in the zerotemperature case cannot be defined uniquely anymore. From a phenomenological standpoint, this implies that the partition function always exhibits a dependence on the chemical potential at finite temperature whereas this is not necessarily the case at $T=0$. There, a dependence is only observed if the chemical potential exceeds the critical value $\mu_{\mathrm{c}}$ determined by the vacuum pole masses and fermion numbers of those fields that are coupled to the chemical potential.

With respect to regulators, we add that the regulator class defined by the relations (14) and (15) also respect the discrete symmetry present at finite temperature. However, identity (20) can no longer be applied to control the regulator-induced explicit breaking of the Silver-Blaze symmetry since it relies on the consideration of infinitesimal transformations.

We close by noting that, from a more general standpoint, we analyzed the properties of $n$-point functions in the complex plane in this section which is also potentially relevant for computations of real-time correlation functions $[35,36,43,53-58]$ within the functional RG framework.

\section{DERIVATIVE EXPANSION AND BCS SCALING}

With the constraints (14) and (15) for regulators respecting the invariance under Silver-Blaze transformations at hand, we now discuss the computation of the effective action in a derivative expansion. In the previous section, we have already pointed out that, in addition to the regulator, the expansion point associated with the derivative expansion has to be chosen carefully in order to ensure that the Silver-Blaze symmetry is not broken explicitly. As we now demonstrate, however, the expansion point also affects the scaling behavior of physical observables. Depending on the chosen expansion point, for example, observables may be found to decrease with increasing chemical potential although an increase may be expected, for example, as is the case in BCS-type models. Moreover, we illustrate that, if Silver-Blaze-symmetric regulators of the type (14) and (15) are used without using a Silver-Blaze-symmetric expansion point, loop integrals may even turn out to be illdefined.

Let us now analyze the derivative expansion of the effective action at finite chemical potential at vanishing temperature. To this end, we employ a simple quarkdiquark model with two quark flavors and three colors for illustration. Its classical action reads

$$
\begin{aligned}
S= & \int \mathrm{d}^{4} x\left\{\bar{\psi}\left(\mathrm{i} \partial+\mathrm{i} \mu \gamma_{0}\right) \psi+\bar{\nu}^{2} \phi_{A}^{*} \phi_{A}\right. \\
& \left.+\mathrm{i} \bar{\psi} \gamma_{5} \tau_{2} \phi_{A}^{*} T^{A} \mathcal{C} \bar{\psi}^{T}-\mathrm{i} \psi^{T} \mathcal{C} \gamma_{5} \tau_{2} \phi_{A} T^{A} \psi\right\} .
\end{aligned}
$$

Here, $\mathcal{C}=\gamma_{2} \gamma_{0}$ is the charge conjugation operator and $\tau_{2}$ is the second Pauli matrix living in flavor space. The fermion fields $\bar{\psi}$ and $\psi$ are understood to contain the two quark flavor degrees of freedom. The sum over the color index $A$ runs only over the antisymmetric color generators $T^{A}$ in the fundamental representation.

The complex-valued scalar fields $\phi_{A}$ carry fermion number $|F|=2$ since they represent diquark states of the form $\phi_{A} \sim\left(\bar{\psi} \gamma_{5} \tau_{2} T^{A} \mathcal{C} \bar{\psi}^{T}\right)$ with $J^{P}=0^{+}$for the total 
angular momentum $J$ and parity $P$. The parameter $\bar{\nu}$ can be viewed as an external "control knob" which can be used to determine the ground state properties of this model in the vacuum $(\mu=0)$. A general fixed-point analysis indeed reveals that two qualitatively distinct ground states are possible, see, e.g., Refs. [23,24,59] and also Ref. [2] for a mean-field analysis. First, the ground state in the vacuum limit is already governed by the formation of a diquark condensate, which breaks the $U_{\mathrm{V}}(1)$ symmetry, and second, the $U_{\mathrm{V}}(1)$ symmetry is only broken at finite $\mu$ because of a Cooper instability in the system but remains intact in the vacuum limit. For the first scenario, we have to choose $\bar{\nu}^{2}$ to be positive but sufficiently small. For the second scenario, we have to choose a sufficiently large value of $\bar{\nu}^{2}$. Thus, a critical value $\bar{\nu}_{*}$ (associated with a non-Gaußian fixed point) must exist which separates these two scenarios from each other.

In the present work, we do not aim at a detailed study of this model. We only employ it to demonstrate issues associated with the Silver-Blaze symmetry which arise in the computation of the effective action at finite chemical potential. To this end, it suffices to compute the effective action of this model in a one-loop approximation where we only take into account purely fermionic loops. This would still allow for a finite running of the wave function renormalization factors of the diquark fields [49]. For simplicity, however, we drop them as well.

In the following, we only consider the RG running of the masslike parameter $\bar{\nu}_{k}^{2}$. Since $\bar{\nu}_{k}^{2}$ determines the curvature of the effective potential at the origin, an analysis of the scale dependence of $\bar{\nu}_{k}^{2}$ allows us to study the onset of the spontaneous breakdown of the $U_{\mathrm{V}}(1)$ symmetry associated with diquark condensation. Indeed, starting the RG flow at a sufficiently large scale $k=\Lambda \gg \mu$ in the $U_{\mathrm{V}}(1)$ symmetric regime, we have $\bar{\nu}_{k}^{2}>0$ at least for a certain range of values of $k \leq \Lambda$. Depending on the initial value $\bar{\nu}_{\Lambda}^{2} \equiv \bar{\nu}^{2}$, the parameter $\bar{\nu}_{k}^{2}$ may then change its sign at a scale $k_{\mathrm{cr}}$, indicating the emergence of a nontrivial ground state of the effective potential and therefore of the spontaneous breakdown of the $U_{\mathrm{V}}(1)$ symmetry, see, e.g., Refs. [23,24,59] for a detailed discussion. We refer to $k_{\mathrm{cr}}$ as the critical scale. This scale sets the scale for all lowenergy quantities $\mathcal{O}$ with mass dimension $d_{\mathcal{O}}$,

$$
\mathcal{O} \sim k_{\mathrm{cr}}^{d_{\mathcal{O}}}
$$

We would like to add that the inverse of $\bar{\nu}_{k}^{2}$ can be traced back to a four-quark interaction associated with a diquark channel. In such a formulation, the onset of spontaneous symmetry breaking is then indicated by a divergence of the corresponding four-quark coupling [23,24,59].

From here on, we assume that the initial condition $\bar{\nu}_{\Lambda}^{2}$ for the RG equation of $\bar{\nu}_{k}^{2}$ has been chosen such that the $U_{\mathrm{V}}(1)$ symmetry remains intact on all scales for $\mu=0$. For finite $\mu$, the emergence of a sign change of $\bar{\nu}_{k}^{2}$ is then solely due to the presence of a Cooper instability, and it was shown [4,5] that the associated critical scale obeys the following scaling behavior:

$$
k_{\mathrm{cr}} \sim \exp \left(-\frac{c}{\mu^{2}}\right)
$$

where $c$ is a dimensionful positive constant which is determined by the initial condition $\bar{\nu}_{\Lambda}^{2}>0$. In the limit $\mu \rightarrow 0$, we have $k_{\text {cr }} \rightarrow 0$; i.e., the $U_{\mathrm{V}}(1)$ symmetry remains intact on all scales. Note that the dependence of $k_{\mathrm{cr}}$ on $\mu$ is handed down to physical observables (e.g., the gap) in the IR limit, leading to the typical exponential scaling behavior in BCS-type theories.

Let us now discuss the RG flow of $\bar{\nu}_{k}^{2}$ and the resulting scaling of the critical scale $k_{\mathrm{cr}}$ with $\mu$ in the light of the Silver-Blaze symmetry. For our concrete calculations, we employ the Wetterich equation [51]. The RG flow equation for $\bar{\nu}_{k}^{2}$ can then be deduced from the scale-dependent twopoint function of the complex scalar fields,

$$
\Gamma_{k, A B}^{(2)}(p, q):=\left.\frac{\delta^{2} \Gamma_{k}\left[\bar{\Psi}_{\mathrm{cl}}, \Psi_{\mathrm{cl}}, \Phi_{\mathrm{cl}}^{*}, \Phi_{\mathrm{cl}}\right]}{\delta \Phi_{\mathrm{cl}, A}^{*}(p) \delta \Phi_{\mathrm{cl}, B}(q)}\right|_{\substack{\bar{\Psi}_{\mathrm{cl}}=\Psi_{\mathrm{cl}}=0 \\ \Phi_{\mathrm{cl}}^{*}=\Phi_{\mathrm{cl}}=0}}
$$

For our discussion, it is convenient to separate the trivial color and momentum dependence of this two-point function from the rest,

$$
\Gamma_{k, A B}^{(2)}(p, q)=\tilde{\Gamma}_{k}^{(2)}(p) \delta_{A B}(2 \pi)^{4} \delta^{(4)}(p+q) .
$$

The derivative expansion of the effective action can be traced back to an expansion of the $n$-point 1PI correlation functions in terms of their external momenta which ultimately requires us to specify an expansion point. As we do not take into account the running of the wave function renormalizations which are obtained by taking derivatives of the two-point function with respect to the external momenta, the scale dependence of the parameter $\bar{\nu}_{k}^{2}$ is simply obtained from an evaluation of the two-point function on the expansion point; i.e., it is associated with the zeroth order of the derivative expansion.

For our computation of the two-point function, we employ a class of regulators which only depend on the spatial momenta but neither on the chemical potential nor on the timelike momentum and therefore satisfy trivially the SilverBlaze constraints (14) and (15). Since we do not take into account diagrams with internal boson lines in our example study, we only have to regularize fermion lines in the loop diagrams. To be specific, we choose the standard form for three-dimensional regulators for fermions [60-63],

$$
R_{k}^{\psi /}=-\vec{p} r\left(\vec{p}^{2} / k^{2}\right)
$$


Here, the so-called regulator shape function $r$ is to a large extent at our disposal [51] and is only specified in concrete evaluations of loop diagrams below. With this class of regulators, we find the following flow equation for the two-point function of the complex scalar fields:

$$
\partial_{t} \tilde{\Gamma}_{k}^{(2)}\left(p_{0}, \vec{p}\right)=-8 \int_{q} \tilde{\partial}_{t} \frac{\left(p_{0}+q_{0}^{-}\right) q_{0}^{+}+(\vec{p}+\vec{q}) \cdot \vec{q}\left(1+r\left((\vec{p}+\vec{q})^{2} / k^{2}\right)\right)\left(1+r\left(\vec{q}^{2} / k^{2}\right)\right)}{\left(\left(p_{0}+q_{0}^{-}\right)^{2}+(\vec{p}+\vec{q})^{2}\left(1+r\left((\vec{p}+\vec{q})^{2} / k^{2}\right)\right)^{2}\right)\left(\left(q_{0}^{+}\right)^{2}+\vec{q}^{2}\left(1+r\left(\vec{q}^{2} / k^{2}\right)\right)^{2}\right)},
$$

where $t=\ln (k / \Lambda)$ is the RG time with $\Lambda$ being the initial RG scale, $\bar{r}_{\vec{q}}=r\left(\vec{q}^{2} / k^{2}\right), q_{0}^{ \pm}=q_{0} \pm \mathrm{i} \mu$, and $\tilde{\partial}_{t}=\left(\partial_{t} r\right) \frac{\partial}{\partial r}$. Note that the integral on the right-hand side is associated with the loop integral depicted in Fig. 1 (left).

Since we have chosen a class of regulators that respects the Silver-Blaze symmetry and we have also not yet chosen an expansion point for the derivative expansion, the RG flow of the fully momentum-dependent two-point function does not violate the Silver-Blaze symmetry. In the spirit of the derivative expansion, we now evaluate the flow equation (33) on a given expansion point. This corresponds to a projection of this flow equation onto the flow equation for the parameter $\bar{\nu}_{k}^{2}$.

We begin by choosing an expansion point which respects the Silver-Blaze symmetry. As discussed in the previous section, this requires us to choose a point in the complex $p_{0}$ plane. A possible choice is $\left(p_{0}=2 \mathrm{i} \mu, \vec{p}=0\right)$. The RG flow equation for $\bar{\nu}_{k}^{2}$ then reads

$$
\begin{aligned}
\partial_{t} \bar{\nu}_{k}^{2} & =\frac{1}{V_{4 d}} \partial_{t} \tilde{\Gamma}_{k}^{(2)}\left(p_{0}=2 \mathrm{i} \mu, \vec{p}=0\right), \\
& =-8 \int_{q} \tilde{\partial}_{t} \frac{1}{\left(q_{0}^{+}\right)^{2}+\vec{q}^{2}\left(1+r\left(\vec{q}^{2} / k^{2}\right)\right)^{2}},
\end{aligned}
$$

where $V_{4 d}=\int \mathrm{d}^{4} x$. Interpreting the right-hand side of this equation in terms of Feynman diagrams, we observe that the integral now corresponds to the loop integral depicted in Fig. 1 (right) when evaluated on vanishing external fourmomentum $\left(p_{0}=0, \vec{p}=0\right)$. In other words, the evaluation of the original loop integral on the Silver-Blaze-symmetric point induces a shift of the chemical potential of one of the internal fermion lines. To analyze the consequences of this
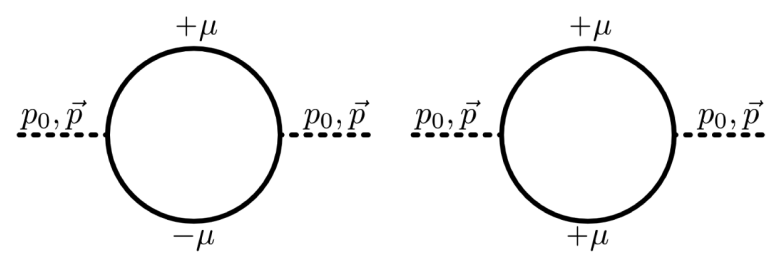

FIG. 1. 1PI diagrams related to the RG flow of the two-point function of the complex scalar field. The dashed lines are associated with the complex scalar field where $\left(p_{0}, \vec{p}\right)$ refers to the external momenta. The solid lines are associated with fermions. shift, we evaluate this loop integral for a given regulator. For convenience, we choose the so-called linear regulator shape function [63],

$$
r_{\operatorname{lin}}(x)=\left(\frac{1}{\sqrt{x}}-1\right) \theta(1-x),
$$

in Eq. (34) and obtain

$$
\partial_{t} \bar{\nu}_{k}^{2}=\frac{2 k^{2}}{3 \pi^{2}} \theta(k-\mu) .
$$

The solution of this equation reads

$$
\bar{\nu}_{k}^{2}=\bar{\nu}_{\Lambda}^{2}-\bar{\nu}_{*}^{2}+\frac{k_{0}^{2}}{3 \pi^{2}},
$$

where $k_{0}=\max (k, \mu)$, and $\bar{\nu}_{*}^{2}$ is the aforementioned critical value for the parameter $\bar{\nu}^{2} \equiv \bar{\nu}_{\Lambda}^{2}>0$. For the regulator shape function (35), we find $\bar{\nu}_{*}^{2}=\Lambda^{2} /\left(3 \pi^{2}\right)$. Choosing $\bar{\nu}_{\Lambda}^{2}>\bar{\nu}_{*}^{2}$, we observe that $\bar{\nu}_{k}^{2}$ remains positive on all scales, and therefore, the $U_{\mathrm{V}}(1)$ symmetry remains intact even in the IR limit. The same is true for $\bar{\nu}_{\Lambda}^{2}=\bar{\nu}_{*}^{2}$ and $\mu=0$, but this choice now defines a strongly interacting scale-invariant point which is only destabilized in the presence of a finite chemical potential. In particular, we observe that there is no finite critical scale $k_{\text {cr }}$ for $\bar{\nu}_{\Lambda}^{2} \geq \bar{\nu}_{*}^{2}$. This implies the absence of dynamical $U_{\mathrm{V}}(1)$ symmetry breaking in this case. As a consequence, the scaling of physical observables with the chemical potential $\mu$ does not agree with the scaling behavior (29). We conclude that a derivative expansion around the Silver-Blaze-symmetric point $\left(p_{0}=\right.$ $2 \mathrm{i} \mu, \vec{p}=0)$ is not suitable to recover the expected BCStype scaling behavior of physical observables.

For $\bar{\nu}_{\Lambda}^{2}<\bar{\nu}_{*}^{2}$, the curvature $\bar{\nu}_{k}^{2}$ of the effective potential may become negative in the RG flow, depending on the actual value of the chemical potential. In fact, for $\mu^{2}<\mu_{0}^{2}=3 \pi^{2}\left(\bar{\nu}_{*}^{2}-\bar{\nu}_{\Lambda}^{2}\right)$, we find $\bar{\nu}_{k=0}^{2}<0$ which indicates that the ground state is governed by spontaneous $U_{\mathrm{V}}(1)$ symmetry breaking for these values of the chemical potential. Note that $\mu_{0}$ defines an upper bound for the critical value $\mu_{\mathrm{c}}$ of the chemical potential introduced in Sec. II. Indeed, the critical scale for $\mu=0$ is given by $k_{\text {cr }}=\sqrt{3 \pi^{2}\left(\bar{\nu}_{*}^{2}-\bar{\nu}_{\Lambda}^{2}\right)}$, and it follows from Eq. (37) that this scale does not depend on the chemical potential, provided 
that we choose $\mu^{2}<k_{\mathrm{cr}}^{2}=\mu_{0}^{2}$. Therefore, all physical observables are expected to be independent of $\mu$ at least for some range of $\mu<\mu_{0}$. In any case, since $k_{\mathrm{cr}} \rightarrow 0$ for $\mu \rightarrow \mu_{0}$, the critical scale eventually decreases (rather than increases) when the chemical potential is increased, in contradistinction to the case of BCS-type scaling, see Eq. (29). This is a severe problem even if we would argue that physical (low-energy) observables, such as the gap, may still exhibit BCS-type scaling behavior although the critical scale does not. In the present case, however, this would imply that the low-energy observables increase continuously with increasing chemical potential while the critical scale decreases and eventually tends to zero above some critical value.

From a phenomenological standpoint, one may argue that the derivative expansion should not be anchored at the Silver-Blaze-symmetric point in the complex $p_{0}$ plane but rather at the conventional point $\left(p_{0}=0, \vec{p}=0\right)$. For the latter point, the flow equation for $\bar{\nu}_{k}^{2}$ is readily obtained from the flow equation (33),

$$
\partial_{t} \bar{\nu}_{k}^{2}=-8 \int_{q} \tilde{\partial}_{t} \mathcal{I}_{k}\left(q_{0}^{+}, q_{0}^{-}, \vec{q}\right),
$$

with

$\mathcal{I}_{k}\left(q_{0}^{+}, q_{0}^{-}, \vec{q}\right)=\frac{q_{0}^{+} q_{0}^{-}+\vec{q}^{2}(1+r)^{2}}{\left(\left(q_{0}^{+}\right)^{2}+\vec{q}^{2}(1+r)^{2}\right)\left(\left(q_{0}^{-}\right)^{2}+\vec{q}^{2}(1+r)^{2}\right)}$.

In terms of Feynman diagrams, the integral on the righthand side is now associated with the diagram depicted in Fig. 1 (left) evaluated on $\left(p_{0}=0, \vec{p}=0\right)$. As shown in Ref. [59], however, a second-order pole at $k=\mu$ is hidden in this loop diagram, rendering the RG flow of the parameter $\bar{\nu}_{k}^{2}$ ill-defined. This is best seen by employing again the regulator shape function (35). The flow equation for $\bar{\nu}_{k}^{2}$ then reads

$$
\partial_{t} \bar{\nu}_{k}^{2}=\frac{k^{4}}{3 \pi^{2}}\left\{\frac{1}{(k+\mu)^{2}}+\frac{\operatorname{sgn}(k-\mu)}{(k-\mu)^{2}}\right\},
$$

which reduces to the flow equation (36) in the limit $\mu \rightarrow 0$ as it should be. From the flow equation (40), we indeed deduce that the RG flow is not well defined when we insist on integrating out fluctuations from $k=\Lambda$ (high-energy scale) to $k=0$ (low-energy limit). This is also true for regulator shape functions other than Eq. (35) and can be traced back to the fact that the conventionally used class of regulators [51,64] defines an RG flow from a given highenergy scale down to the low-energy limit. Thus, the aforementioned singularity at $k=\mu$ is always approached from one side. The actual appearance of this divergence has its origin in the Cooper instability which is of course not pathologic at all. Only the "treatment" of this divergence with the aid of conventional regulator classes is problematic.

One may now be tempted to argue that this singularity is lifted for any (even infinitesimally) finite temperature $T$ [59], and in practice, one should only consider flows at finite temperature and extrapolate to the zero-temperature limit afterwards. However, this does not cure the actual problem of having an ill-defined flow at zero temperature, and therefore, this idea should be discarded. In this respect, we also note that the parameters of models are usually determined at $T=0$.

Leaving the finite-temperature case aside, one may argue that the divergence in the flow is cured by the presence of a finite diquark gap (i.e., BCS-type gap, in general). However, this requires that the gap has already been generated in the RG flow at a scale $k_{\mathrm{cr}}>\mu$, i.e., before the RG flow "hits" the divergence at $k=\mu$. Apparently, this involves a tuning of the parameters of the model. In particular, it excludes to study the case where, for example, the parameters are chosen such that the $U_{\mathrm{V}}(1)$ symmetry of the theory remains intact on all scales for $\mu=0$. Therefore, also this "strategy" of tuning the parameters of the model should be only considered with great care, if at all, since the underlying RG flow is still not well defined. We add that, in first-principles studies of QCD, a tuning of parameters, such as $\bar{\nu}_{\Lambda}^{2}$, is not even possible since $\bar{\nu}_{\Lambda}^{2}$ is initially zero and solely generated by quark-gluon interactions in the form of four-quark interaction channels, see also Ref. [24].

From our discussion of the flow equation (40), we can now deduce how the divergence in the RG flow can be "cured", namely, by gapping fluctuations around the Fermi surface with the aid of an artificial gap. The latter should then be successively removed towards the end point of the RG flow. This requires to construct a regulator that integrates out fluctuations around the Fermi surface and thereby introduces a gap for fluctuations at the Fermi surface. Let us give a qualitative illustration for such a prescription by means of the flow equation (40). Formally, the solution of this $\mathrm{RG}$ equation reads ${ }^{1}$

$$
\bar{\nu}_{k}^{2}-\bar{\nu}_{\Lambda}^{2} \sim \int_{\Lambda}^{k} \mathrm{~d} k^{\prime} k^{\prime 3} \frac{\operatorname{sgn}\left(k^{\prime}-\mu\right)}{\left(k^{\prime}-\mu\right)^{2}}
$$

Here, we have dropped prefactors, and the contribution analytic at $k=\mu$ as the latter is irrelevant for our qualitative discussion at this point. We now employ a principal value prescription to compute this integral which mimics the effect of the implementation of a sharp cutoff around the Fermi scale $\mu$,

\footnotetext{
${ }^{1}$ Note that, in more elaborate studies, the right-hand side of the flow equation (40) also depends on other couplings (e.g., Yukawa-type and four-diquark couplings) which hinders a direct integration of the flow equation.
} 
$\bar{\nu}_{0}^{2}(\varepsilon)-\bar{\nu}_{\Lambda}^{2} \sim-\int_{\mu-\varepsilon}^{0} \mathrm{~d} k^{\prime} \frac{k^{\prime 3}}{\left(k^{\prime}-\mu\right)^{2}}+\int_{\Lambda}^{\mu+\varepsilon} \mathrm{d} k^{\prime} \frac{k^{\prime 3}}{\left(k^{\prime}-\mu\right)^{2}}$.

To obtain this equation, we have set $k=0$ in Eq. (41). Note that IR divergences as they may occur for $\mu=0$ are screened anyhow by the presence of the chemical potential. Moreover, we have introduced a new scale $\varepsilon$ by hand which plays the role of the former RG scale $k .^{2}$ In the limit $\varepsilon \rightarrow 0$, we then recover Eq. (41) for $k \rightarrow 0$. In any case, performing the integration in Eq. (42), we find $\bar{\nu}_{0}^{2}(\varepsilon)-\bar{\nu}_{\Lambda}^{2} \sim \mu^{2} \ln \varepsilon$, where we dropped nondivergent terms for $\varepsilon \rightarrow 0$ as well as prefactors that are irrelevant for our line of arguments. Using that $\bar{\nu}_{0}^{2}\left(\varepsilon=\varepsilon_{\mathrm{cr}}\right)=0$ at the critical scale $\varepsilon_{\mathrm{cr}}$ associated with $U_{\mathrm{V}}(1)$ symmetry breaking, we find $\varepsilon_{\text {cr }} \sim \exp \left(-c / \mu^{2}\right)$. Here, the constant $c>0$ is related to the parameter $\bar{\nu}_{\Lambda}^{2}$. Thus, in line with our discussion of Eq. (29), we expect the typical BCS-type scaling behavior of low-energy observables with the chemical potential since the scale for the latter is set by the scale $\varepsilon_{\mathrm{cr}}$. Note that in our construction of a new regulator class in Sec. IV, the RG scale $k$ effectively plays the role of the scale $\varepsilon$ and should therefore not be confused with the RG scale $k$ introduced by conventional regulators, at least for finite $\mu$.

The BCS-type behavior has indeed been revealed in Refs. [4,5] by implementing a sharp cutoff around the Fermi scale $\mu$ to integrate out fluctuations around the Fermi surface rather than following the RG flow of the theory from a high-energy scale down to a low-energy scale. With respect to the functional RG approach, we have so far only implemented this idea by hand for illustrational purposes. In order to do this in a systematic fashion that is readily generalizable to approximations more involved than the one considered in this section, we construct a suitable class of fermion regulators in the subsequent section. The class is very general in the sense that it does not require the use of a sharp cutoff which often comes at a price of nonlocality [64] and also ambiguities in the actual computation of RG flow equations, see, e.g., Ref. [65]. For nonrelativistic theories, such a class of regulators has already been constructed within the functional RG framework and successfully employed to study a variety of systems, ranging from condensed-matter systems to ultracold atomic gases [26-28,66-69], see Ref. [70] for a discussion of optimization of RG flows in this context. Unfortunately, a naive generalization of these regulators to relativistic theories is already hindered by the chiral symmetry. An additional constraint for our regulator construction is set by the fact that we would like to ensure that this new class of regulators

\footnotetext{
${ }^{2}$ Note that this statement has to be taken with some care since we do not recover Eq. (41) for $\varepsilon \rightarrow \Lambda$. In fact, there is no simple map between the scales $k$ and $\varepsilon$. Therefore, we restrict ourselves to $\varepsilon \ll \mu<\Lambda$ which is sufficient for our line of arguments here.
}

reduces to the class of conventionally used regulators which regularize divergences in the low-momentum limit.

Already at this point, we would like to emphasize that it comes at a price to integrate out fluctuations around the Fermi surface. Loosely speaking, the implementation of this idea requires to couple the spatial momenta to the chemical potential which then unavoidably leads to a breaking of the Silver-Blaze symmetry. However, as discussed above, we require to break this symmetry anyhow in a derivative expansion by choosing a suitable expansion point in order to recover the correct long-range behavior of physical observables, i.e., BCS-type scaling. In any case, by choosing a regulator which breaks the Silver-Blaze symmetry, the RG flow violates the Silver-Blaze symmetry, and therefore, also the end point of the RG flow (IR limit, $k \rightarrow 0$ ) still "knows" about this violation, although the regulator vanishes in the IR limit. This is true even if a Silver-Blaze symmetric expansion point is chosen in momentum space. Indeed, a similar situation is encountered when three-dimensional regulators are used for studies of theories in the vacuum limit. Although the regulator vanishes in the IR limit, the wave function renormalization factors associated with timelike and spacelike derivatives differ in the limit $k \rightarrow 0$, indicating the breaking of the Lorentz symmetry induced by the regulator in the RG flow, see, e.g., Ref. [71] for a discussion. Moreover, this issue is also encountered in functional RG studies of gauge theories. There, it is required to take into account modified Ward-Takahashi identities in order to take care of the gauge-symmetry breaking induced by the regulator in the RG flow. Otherwise, the IR results may suffer from gauge-symmetry violating contributions, even though the regulator vanishes in the limit $k \rightarrow 0$, see, e.g., Refs. [45,72,73] for reviews.

\section{RG FLOWS AROUND THE FERMI SURFACE}

\section{A. Chiral fermions}

For our construction of a regulator suitable to deal with fermions in the presence of a Cooper instability, it is convenient to define the following two projectors:

$$
P_{ \pm} \equiv P_{ \pm}(\vec{p})=\frac{1}{2 \mathrm{i}}\left(\mathrm{i} \gamma_{0} \pm \frac{\vec{p}}{|\vec{p}|}\right) \gamma_{0}
$$

We have

$$
\begin{gathered}
P_{+}+P_{-}=\mathbb{1}, \quad P_{+} P_{-}=P_{-} P_{+}=0, \\
P_{+} P_{+}=P_{+}, \quad P_{-} P_{-}=P_{-},
\end{gathered}
$$

and

$$
P_{ \pm} \gamma_{0}=\gamma_{0} P_{\mp}, \quad\left\{P_{ \pm}, \gamma_{0}\right\}=\gamma_{0} .
$$


Note also that $P_{ \pm}(\vec{p})=P_{\mp}(-\vec{p})$. Basically, the operators $P_{ \pm}$are projection operators on positive and negative energy solutions of the free Dirac equation, thus associated with particle and antiparticle states. Similar forms of these projectors are often introduced in quantum field theory textbooks (see, e.g., Refs. [74,75]) and are also employed in hard dense loop studies (see, e.g., Ref. [76]). Phenomenologically speaking, the fact that

$$
\operatorname{tr} P_{ \pm}=2
$$

reminds us that the free Dirac equation comes with two solutions with positive energy and two solutions with negative energy.

In Eq. (1), the kinetic term $S_{\bar{\psi} \psi}$ for the fermions reads

$$
S_{\bar{\psi} \psi}=\int_{p} \bar{\Psi}\left\{-\left(p_{0}-\mathrm{i} \mu\right) \gamma_{0}-\vec{p}\right\} \Psi
$$

where $\bar{\Psi}$ and $\Psi$ are the Fourier transforms of the fields $\bar{\psi}$ and $\psi$, respectively. For convenience, we define the kinetic operator $T$,

$$
T=-\left(p_{0}-\mathrm{i} \mu\right) \gamma_{0}-\vec{p} .
$$

With the aid of the projectors $P_{ \pm}$, we can decompose $T$ as follows:

$$
T=C_{-} P_{-} \gamma_{0}+C_{+} P_{+} \gamma_{0},
$$

where

$$
C_{\mp}=-p_{0}-\mathrm{i}(-\mu \mp|\vec{p}|) .
$$

Using this decomposition, the kinetic term $S_{\bar{\psi} \mu}$ for the fermions can be split into two parts,

$$
\int_{p} \bar{\Psi} C_{-} P_{-} \gamma_{0} \Psi, \quad \text { and } \quad \int_{p} \bar{\Psi} C_{+} P_{+} \gamma_{0} \Psi .
$$

We observe that these two terms are separately invariant under chiral transformations. Moreover, both terms are also separately invariant under Silver-Blaze transformations. Under charge conjugation, the two terms are not invariant. For $\mu=0$, however, they can still be transformed into each other under charge conjugation. Phenomenologically speaking, this is a consequence of the fact that the projectors $P_{ \pm}$decompose the kinetic term $S_{\bar{\psi} \psi \psi}$ into particle and antiparticle contributions. Only $\int_{p} \bar{\Psi} T \Psi$ is invariant under the charge conjugation for $\mu=0$. Note that the symmetry under charge conjugation is broken explicitly by the presence of a finite chemical potential.

Using the properties (44)-(46) of the projectors $P_{ \pm}$, the inverse of the operator $T$-which basically appears in the computation of loop diagrams-is readily constructed,

$$
T^{-1}=C_{-}^{-1} P_{+} \gamma_{0}+C_{+}^{-1} P_{-} \gamma_{0} .
$$

The physical meaning of $C_{ \pm}$becomes apparent when we switch to Minkowski spacetime, $p_{0} \rightarrow-\mathrm{i} p_{0}$. We then observe that $T^{-1}$ exhibits poles at $p_{0}=\omega_{ \pm}$with

$$
\omega_{ \pm}= \pm|\vec{p}|-\mu
$$

The positions of the poles define nothing but the dispersion relation of relativistic (quasi)particles in the presence of a chemical potential; i.e., it is essentially the energy of the (anti)fermions relative to the Fermi surface located at $|\vec{p}|=\mu$.

We now switch back to Euclidean spacetime and note that the inversion of $T$ has to be considered with care. In fact, whereas $C_{-}$is invertible since $C_{-} \neq 0$ for any finite $\mu$, this is not the case for $C_{+}$. The latter vanishes for $p_{0}=0$ and $|\vec{p}|=\mu$ (i.e., at the Fermi surface), and strictly speaking, $T$ is therefore not always invertible. At vanishing chemical potential, this singular point corresponds to the case of vanishing four-momentum of which is then taken care by, for example, employing a masslike regularization scheme. In the presence of a finite chemical potential, however, the use of such a scheme does in general not "cure" the divergence at the Fermi surface, and the divergence associated with vanishing four-momentum is screened by the chemical potential anyhow.

Let us continue with the construction of a class of regulators which is capable of handling the (quasi)particle dispersion relations in a suitable manner. Our starting point is Eq. (50), i.e., the decomposition of the kinetic operator $T$ into positive and negative energy solutions relative to the Fermi surface. The basic idea is now to treat the modes associated with the two terms as two types of modes which are regularized differently in the presence of a finite chemical potential ${ }^{3}$ but still regularized in the same way in the limit of vanishing chemical potential. The latter requirement ensures that modes with positive and negative energy are treated in the same way for $\mu=0$, and the regularized kinetic term does therefore not break explicitly the charge conjugation symmetry in this limit. In any case, we also require that the regulator does not break the chiral symmetry of the theory under consideration. A general regulator for fermions fulfilling these requirements can be written in the following form:

$R_{k}^{\psi}=-\mathrm{i}(-\mu-|\vec{p}|) r_{-} P_{-} \gamma_{0}-\mathrm{i}(-\mu+|\vec{p}|) r_{+} P_{+} \gamma_{0}$,

where $r_{ \pm}$are again dimensionless regulator shape functions. From here on, we assume that these functions are of the following form:

\footnotetext{
${ }^{3}$ Note that the chemical potential indeed allows us to distinguish the two types of modes as it breaks the charge conjugation symmetry.
} 


$$
r_{ \pm}:=r\left(x_{ \pm}\right)
$$

where

$$
x_{ \pm} k^{2}=(-\mu \pm|\vec{p}|)^{2} .
$$

Note that we use the same functional form $r$ for the regulator of the two modes which ensures that we recover the standard form of the flow equation in the absence of a chemical potential (where both types of modes are then treated identically). The use of different functional forms would instead imply that the charge conjugation symmetry is broken explicitly by the regularization scheme even in the limit $\mu \rightarrow 0$. In any case, the form of the regulator proposed in Eq. (55) already breaks the Silver-Blaze symmetry by construction which, however, appears necessary in a derivative expansion of the effective action in order to recover the expected BCS scaling, see Sec. III.

Regarding the shape functions $r$, we, in general, assume that $(1+r) \geq 0$ and that they obey

$$
\lim _{x \rightarrow 0} \sqrt{x} r(x)>0
$$

as well as

$$
\lim _{x \rightarrow \infty} r(x)=0 .
$$

For example, the property (58) implies that

$$
r\left(x_{+}\right)=\frac{k}{|\mu-| \vec{p}||}+\ldots
$$

Loosely speaking, this ensures that, in the RG flow, fluctuations at the Fermi surface are gapped. The property (59) ensures that the regulator vanishes in the limit of $k \rightarrow 0$ for fixed momentum as well as in the limit $|\vec{p}| \rightarrow \infty$ for fixed RG scale $k$. Finally, we add that the property $(1+r) \geq 0$ for any value of $x$ is required since loop diagrams may otherwise be plagued by artificial divergences.

Possible choices for the shape function are given by Eq. (35) and

$$
r_{\exp }(x)=-1+\frac{1}{\sqrt{1-\mathrm{e}^{-x}}},
$$

as well as a polynomial version,

$$
r_{\mathrm{p}}(x)=-1+\frac{1}{\sqrt{1-\left(\sum_{n=0}^{N} \frac{1}{n !} x^{n}\right)^{-1}}},(N>2) .
$$

Finally, one may also define an ordinary sharp cutoff with the aid of the shape function. ${ }^{4}$ Note that the general form of

\footnotetext{
${ }^{4}$ The functional forms are simply adapted from those in Refs. [53,62-64,77-82] introduced for the conventionally used regulator class.
}

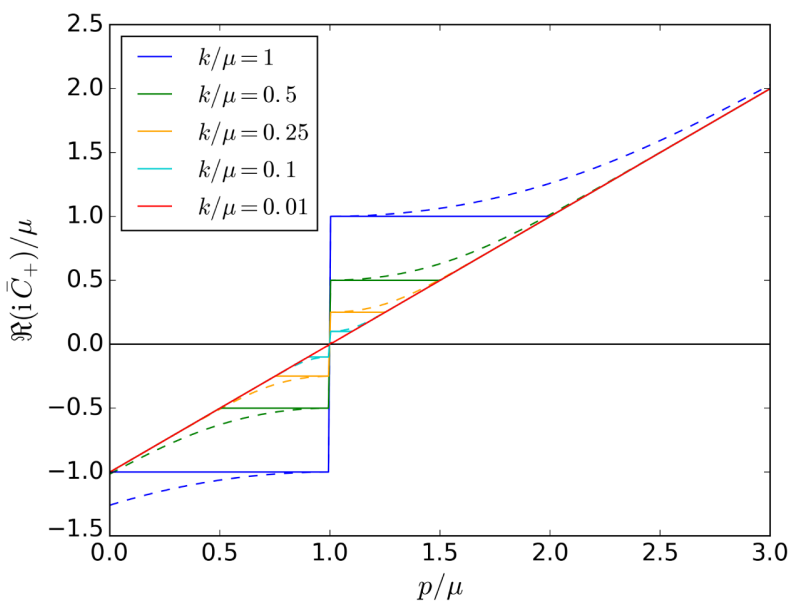

FIG. 2. $\Re\left(\mathrm{i} \bar{C}_{+}\right) / \mu$ as a function of $p / \mu$ for various values of $k / \mu$ as obtained from employing the shape function (35) (solid lines) and the shape function (62) for $N=4$ (dashed lines).

the regulator $R_{k}^{\psi}$ together with any of the listed shape functions is not necessarily optimized in the spirit of Refs. [64,80-82]. In fact, such an optimization of RG flows in the presence of a finite quark chemical potential is beyond the scope of this work. The explicit forms of the shape function are presented here only for illustrational purposes.

By adding the regulator $R_{k}^{\psi /}$ to the kinetic operator $T$, we find

$$
T+R_{k}^{\psi}=\bar{C}_{-} P_{-} \gamma_{0}+\bar{C}_{+} P_{+} \gamma_{0},
$$

where we have introduced regularized "quasiparticle dispersion relations",

$$
\bar{C}_{\mp}=-p_{0}-\mathrm{i}(-\mu \mp|\vec{p}|)\left(1+r_{\mp}\right) .
$$

Using the properties (44)-(46) of the projectors $P_{ \pm}$, the regularized kinetic operator $T+R_{k}^{\psi}$ is readily inverted which is now well defined for all $p_{0}$ and $\vec{p}$. The inversion yields the regularized propagator,

$$
\left(T+R_{k}^{\psi}\right)^{-1}=\bar{C}_{-}^{-1} P_{+} \gamma_{0}+\bar{C}_{+}^{-1} P_{-} \gamma_{0} .
$$

We note that

$$
\Re\left(\mathrm{i} \bar{C}_{+}\right)=(|\vec{p}|-\mu)\left(1+r_{+}\right)
$$

is not positive definite but changes its sign at the Fermi surface. In fact, we have $\Re\left(\mathrm{i} \bar{C}_{+}\right)>0$ for $|\vec{p}|>\mu$ and $\Re\left(\mathrm{i} \bar{C}_{+}\right)<0$ for $|\vec{p}|<\mu$, see also Fig. 2. Close to the Fermi surface, we find

$$
\Re\left(\mathrm{i} \bar{C}_{+}\right)=k \operatorname{sgn}(|\vec{p}|-\mu)+\ldots,
$$

implying that $\Re\left(\mathrm{i} \bar{C}_{+}\right)$is discontinuous at the Fermi surface. In any case, from Eq. (67), we deduce that the regulator 
effectively introduces a gap $\sim k$ for fluctuations around the Fermi surface. By construction, this gap disappears in the limit $k \rightarrow 0$, i.e., in the long-range limit. Hence, fluctuations are integrated out around the Fermi surface. For the negative-energy modes associated with $\bar{C}_{-}$, we note that

$$
\Re\left(\mathrm{i} \bar{C}_{-}\right)<0,
$$

even for $k \rightarrow 0$ because of the presence of the chemical potential which, loosely speaking, acts as a regulator for these modes. ${ }^{5}$

Before we analyze the regularized kinetic operator $T+$ $R_{k}^{\psi}$ in the vacuum limit, we would like to give a useful relation for the evaluation of the Wetterich equation when our present regularization scheme is employed. It reads

$$
\begin{aligned}
& \left(T+R_{k}^{\psi}\right)^{-1}\left(\partial_{t} R_{k}^{\psi}\right) \\
& \quad=\mathrm{i}(\mu+|\vec{p}|) \bar{C}_{-}^{-1}\left(\partial_{t} r_{-}\right) P_{+}+\mathrm{i}(\mu-|\vec{p}|) \bar{C}_{+}^{-1}\left(\partial_{t} r_{+}\right) P_{-} .
\end{aligned}
$$

We observe that the derivatives of the shape functions, which specify the Wilsonian momentum-shell integrations, only appear together with the corresponding "propagators" $\sim \bar{C}_{ \pm}^{-1}$. With the aid of Eq. (47), the trace over Dirac indices appearing in the Wetterich equation can be computed straightforwardly. We find

$$
\begin{aligned}
\operatorname{Tr}_{D}\left\{\left(T+R_{k}^{\psi}\right)^{-1}\left(\partial_{t} R_{k}^{\psi}\right)\right\} \\
\quad=2 \mathrm{i}(\mu+|\vec{p}|) \bar{C}_{-}^{-1}\left(\partial_{t} r_{-}\right)+2 \mathrm{i}(\mu-|\vec{p}|) \bar{C}_{+}^{-1}\left(\partial_{t} r_{+}\right) .
\end{aligned}
$$

It is also worthwhile to note that the $p_{0}$ integration can be performed very efficiently with this decomposition using Cauchy's residue theorem since the poles in the complex $p_{0}$ plane are readily read off from Eq. (70).

With the class of regulators (55) at hand, we can now, in principle, compute loop diagrams and study the RG flow of couplings. Before we illustrate this in Sec. V, we would like to emphasize again that our class of regulators does not violate chiral symmetry and state that, by the proposed

\footnotetext{
${ }^{5}$ One may be tempted to insert only a regulator for the modes associated with $\bar{C}_{+}$and just let the chemical potential regularize the modes with negative energies. However, as indicated above, the vacuum limit $(\mu \rightarrow 0)$, where both types of modes need to be regularized, is then no longer well defined. A still valid alternative in line with our requirements is to consider a regulator of the following form:$$
R_{k}^{\psi}=-\mathrm{i}\left(-\mu-|\vec{p}| r_{-}\right) P_{-} \gamma_{0}-\mathrm{i}(-\mu+|\vec{p}|) r_{+} P_{+} \gamma_{0},
$$

where the argument of the shape function associated with the negative-energy modes is now chosen to be independent of the chemical potential, $r_{-}=r\left(\vec{p}^{2} / k^{2}\right)$, but the functional form is still assumed to be the same for both types of modes.
}

decomposition of the regulator and the inclusion of the chemical potential, we effectively perform a suitable spectral adjustment of the regulator functions.

Let us finally show that we recover the form of conventionally used three-dimensional regulators in the vacuum limit, i.e., $\mu \rightarrow 0[62,63]$. To this end, we consider the regularized kinetic term (63) for $\mu=0 .^{6}$ Looking at the projectors $P_{ \pm}$, we observe that they only depend on the spatial momenta in such a way that they are invariant under a rescaling of the spatial momenta with a positive factor,

$$
P_{ \pm}(\vec{p}(1+r))=P_{ \pm}(\vec{p})
$$

Recall also that $(1+r)>0$ for $k>0$. Thus, formally, we can rescale the spatial momenta in Eq. (63) in this way; i.e., we may set $\vec{p}_{r}:=\vec{p}(1+r)$ and then obtain the following expression for $\mu=0$ by employing again the properties of the projectors $P_{ \pm}$,

$$
T+R_{k}^{\psi r}=-p_{0} \gamma_{0}-\vec{p}_{r}=-p_{0} \gamma_{0}-\vec{p}(1+r) .
$$

This is nothing but the conventional form of the regularized kinetic operator (for a three-dimensional regulator) $[62,63]$. However, we emphasize that, at finite chemical potential, our present regulator is different from this conventional choice since it involves the chemical potential and therefore treats the modes above and below the Fermi surface in a different way.

Although our proposed regulator (55) comes with many advantages, it should be stated that it breaks the SilverBlaze symmetry explicitly, ${ }^{7}$ in contrast to conventional three-dimensional regulator functions. However, the latter class of regulators leads to ill-defined loop diagrams because of an insufficient treatment of the Cooper instability, see our discussion in Sec. III. Moreover, we would like to remind the reader that, in a derivative expansion corresponding to an expansion of correlation functions in external momenta around a given point in momentum space, we require to break the Silver-Blaze symmetry explicitly anyhow by choosing a suitable expansion point such that the correct BCS scaling of observables $\mathcal{O}$ as a function of the chemical potential is recovered, $\mathcal{O} \sim$ $\exp \left(-c / \mu^{2}\right)$ with $c>0$, see Sec. III.

\section{B. Massive fermions}

The regulator class constructed in the previous section appears fine for chiral fermions. Indeed, it respects chiral symmetry and treats modes close to the Fermi surface in an adequate manner. However, it is not appropriate to regularize modes with a finite mass close to the Fermi surface. It should be noted that, unlike IR divergences in the limit

\footnotetext{
${ }^{6}$ Note that $r_{+}=r_{-}=r$ in this case.

${ }^{7}$ In fact, the class of regulators defined in Eq. (55) does not obey the condition (14).
} 
$\mu \rightarrow 0$, divergences at the Fermi surface are not screened by a fermion mass term. Therefore, conventional masslike regulators $[62,63,77]$ are not suitable to deal with such divergences appearing at finite $\mu$. Indeed, a fermion mass (which may be a parameter or may be generated dynamically by interactions, for example, by spontaneous chiral symmetry breaking) rather deforms the Fermi surface. This deformation effectively leads to a shift of the Cooper instability in momentum space. A suitable regulator scheme needs to account for this shift.

Let us begin our construction of a regulator for massive fermions by enhancing our projectors $P_{ \pm}$,

$$
P_{ \pm}(\vec{p}, m)=\frac{1}{2 \mathrm{i}}\left(\mathrm{i} \gamma_{0} \pm \frac{\vec{p}+\mathrm{i} m}{\epsilon}\right) \gamma_{0},
$$

where

$$
\epsilon=\sqrt{\vec{p}^{2}+m^{2}} .
$$

From these new projectors, we recover the projectors defined in Eq. (43) in the limit $m \rightarrow 0$. We again have

$$
\begin{gathered}
P_{+}+P_{-}=\mathbb{1}, \quad P_{+} P_{-}=P_{-} P_{+}=0, \\
P_{+} P_{+}=P_{+}, \quad P_{-} P_{-}=P_{-},
\end{gathered}
$$

and

$$
\operatorname{tr} P_{ \pm}=2
$$

For what follows, it is convenient to define a second set of projectors,

$$
\bar{P}_{ \pm}(\vec{p}, m)=\frac{1}{2 \mathrm{i}}\left(\mathrm{i} \gamma_{0} \pm \frac{\vec{p}-\mathrm{i} m}{\epsilon}\right) \gamma_{0}
$$

Also here, we have

$$
\begin{gathered}
\bar{P}_{+}+\bar{P}_{-}=\mathbb{1}, \quad \bar{P}_{+} \bar{P}_{-}=\bar{P}_{-} \bar{P}_{+}=0, \\
\bar{P}_{+} \bar{P}_{+}=\bar{P}_{+}, \quad \bar{P}_{-} \bar{P}_{-}=\bar{P}_{-},
\end{gathered}
$$

and

$$
\operatorname{tr} \bar{P}_{ \pm}=2
$$

Note also that $\bar{P}_{ \pm}(\vec{p}, m)=\bar{P}_{\mp}(-\vec{p},-m)$. The two sets of projectors are related. First of all, we have $\bar{P}_{ \pm}(\vec{p}, 0)=P_{ \pm}(\vec{p}, 0)$. Moreover, we find

$$
\bar{P}_{+} \gamma_{0}=\gamma_{0} P_{-}, \quad \bar{P}_{-} \gamma_{0}=\gamma_{0} P_{+} .
$$

Finally, we note that

$$
\bar{P}_{ \pm}^{\dagger}=\bar{P}_{ \pm}, \quad P_{ \pm}^{\dagger}=P_{ \pm} .
$$

With these operators, we can now construct a regulator for massive fermions which allows us to integrate out fluctuations around the Fermi surface. To this end, we first add a mass term to the kinetic operator $T$ defined in Eq. (49),

$$
T(m)=-\left(p_{0}-\mathrm{i} \mu\right) \gamma_{0}-\vec{p}+\mathrm{i} m .
$$

This operator can be decomposed as follows:

$$
T(m)=C_{-} \bar{P}_{-} \gamma_{0}+C_{+} \bar{P}_{+} \gamma_{0},
$$

where

$$
C_{\mp} \equiv C_{\mp}(m)=-p_{0}-\mathrm{i}(-\mu \mp \epsilon) .
$$

The inverse of $T(m)$ reads $^{8}$

$$
T^{-1}(m)=C_{-}^{-1} P_{+} \gamma_{0}+C_{+}^{-1} P_{-} \gamma_{0} .
$$

Here, we have used Eq. (82).

A regulator accounting for the fact that the Fermi surface is effectively deformed in the presence of a finite fermion mass is now readily constructed,

$R_{k}^{\psi \prime}(m)=-\mathrm{i}(-\mu-\epsilon) r_{-} \bar{P}_{-} \gamma_{0}-\mathrm{i}(-\mu+\epsilon) r_{+} \bar{P}_{+} \gamma_{0}$.

Examples for possible shape functions $r_{ \pm}=r\left(x_{ \pm}\right)$are given in Eqs. (35), (61), and (62), see also Eq. (56) and our discussion thereof. However, we now define $x_{ \pm}$as follows:

$$
x_{ \pm} k^{2}=(-\mu \pm \epsilon)^{2}
$$

Note that the mass enters the regulator function. This is required since, unlike ordinary IR divergences in the limit $\mu \rightarrow 0$, the Cooper instability is not cured by the presence of a finite fermion mass.

Let us now add the regulator $R_{k}^{\psi}$ to the kinetic operator $T(m)$. We find

$$
T(m)+R_{k}^{\psi}=\bar{C}_{-} \bar{P}_{-} \gamma_{0}+\bar{C}_{+} \bar{P}_{+} \gamma_{0},
$$

where

$$
\bar{C}_{\mp} \equiv \bar{C}_{\mp}(m)=-p_{0}-\mathrm{i}(-\mu \mp \epsilon)\left(1+r_{\mp}\right) .
$$

Using our relations above, the regularized kinetic operator is readily inverted. The inversion yields

$$
\left(T(m)+R_{k}^{\psi}\right)^{-1}=\bar{C}_{-}^{-1} P_{+} \gamma_{0}+\bar{C}_{+}^{-1} P_{-} \gamma_{0} .
$$

\footnotetext{
${ }^{8}$ Of course, the inversion is again not well defined for $p_{0}=0$ and $\epsilon=\mu$. This is taken care of below by inserting a suitable regulator.
} 
As discussed above for the massless limit, the Cooper instability is now also regularized for finite fermion masses. Indeed, close to the Fermi surface, we find for the modes associated with $\bar{C}_{+}$that

$$
\Re\left(\mathrm{i} \bar{C}_{+}\right)=k \operatorname{sgn}(\epsilon-\mu)+\ldots,
$$

implying that the regulator effectively introduces a gap $\sim k$ for massive fermions around the Fermi surface.

Note that the statements regarding the decomposition of $\left(T+R_{k}^{\psi}\right)^{-1}\left(\partial_{t} R_{k}^{\psi}\right)$ made in Eq. (69) for the massless case also apply to the present case of finite fermion masses, $\left(T(m)+R_{k}^{\psi}\right)^{-1}\left(\partial_{t} R_{k}^{\psi}\right)$.

Finally, we would like to add that, even in the limit $\mu \rightarrow 0$, the class of regulators defined by Eq. (88) does not fall into the class of conventionally employed three-dimensional regulators. In fact, considering $\mu=0$ and using ${ }^{9}$

$$
\bar{P}_{ \pm}(\vec{p}(1+r), m(1+r))=\bar{P}_{ \pm}(\vec{p}, m),
$$

we find

$$
T(m)+R_{k}^{\psi}=-p_{0} \gamma_{0}-(\vec{p}-\mathrm{i} m)(1+r),
$$

with a regulator shape function depending on $\left(\vec{p}^{2}+m^{2}\right) / k^{2}$ rather than only on $\vec{p}^{2} / k^{2}$ as in the case of conventionally employed three-dimensional regulators. Nevertheless, our present class of regulators is also a valid class in the limit $\mu \rightarrow 0$ in the presence of a finite mass. Indeed, it rather falls into the class of spectrally adjusted regulators. The latter class is often employed in studies of gauge theories, see, e.g., Refs. [64,73,83-86]. In this spirit, we close this section by noting that $m$ does not have to be a mass in the narrower sense of the word. For example, $m$ may also be related to a chiral (background) field $\chi, m^{2} \sim \chi^{2}$. Moreover, if the running of, for example, wave function renormalization factors is taken into account in a given study, the inclusion of the latter in our proposed class of regulators (i.e., also into the shape function) may be required in order to ensure that the regulator indeed gaps fluctuations around the Fermi surface.

\section{EXAMPLE: DIQUARK CONDENSATION}

We now demonstrate the application of the class of regulators defined in Eq. (55). To this end, we consider again the simple quark-diquark model introduced in Eq. (27) and compute the flow of the parameter $\bar{\nu}_{k}^{2}$ and the four-diquark coupling $\bar{\lambda}_{k}$. Although the latter is set to zero in the classical action, it is induced in the RG flow by quark-diquark interactions. We add that the couplings $\bar{\nu}_{k}^{2}$ and $\bar{\lambda}_{k}$ should be viewed as the zeroth order terms of an expansion of the two-point and four-point functions in their external momenta, respectively. The expansion point is

\footnotetext{
${ }^{9}$ Recall that $x_{+}=x_{-}=x=\epsilon^{2} / k^{2}$ for $\mu=0$.
}

given by $\left(p_{0}, \vec{p}\right)=(0,0)$. For simplicity, we only take into account purely fermionic loops in the present example study, as also done in Sec. III. For details on the derivation of the flow equations presented below, we refer the reader to Appendix B.

The initial condition of the RG flow is given by the classical action (27) which is invariant under $U_{\mathrm{V}}(1)$ transformations. For the $U_{\mathrm{V}}(1)$-symmetric regime, we then find the following set of flow equations:

$$
\begin{aligned}
\partial_{t} \bar{\nu}_{k}^{2}= & -4 \int_{p} \tilde{\partial}_{t}\left(\frac{1}{p_{0}^{2}+(\mu-|\vec{p}|)^{2}\left(1+r_{+}\right)^{2}}\right. \\
& \left.+\frac{1}{p_{0}^{2}+(\mu+|\vec{p}|)^{2}\left(1+r_{-}\right)^{2}}\right),
\end{aligned}
$$

and

$$
\begin{aligned}
\partial_{t} \bar{\lambda}_{k}= & 2 \int_{p} \tilde{\partial}_{t}\left(\left(\frac{1}{p_{0}^{2}+(\mu-|\vec{p}|)^{2}\left(1+r_{+}\right)^{2}}\right)^{2}\right. \\
& \left.+\left(\frac{1}{p_{0}^{2}+(\mu+|\vec{p}|)^{2}\left(1+r_{-}\right)^{2}}\right)^{2}\right),
\end{aligned}
$$

where $\tilde{\partial}_{t}=\left(\partial_{t} r_{+}\right) \frac{\partial}{\partial r_{+}}+\left(\partial_{t} r_{-}\right) \frac{\partial}{\partial r_{-}}$. We observe that the two flow equations are decoupled within our present approximation in the $U_{\mathrm{V}}(1)$-symmetric regime.

From the equation for $\bar{\nu}_{k}^{2}$, we can already extract the scaling behavior of the critical scale $k_{\mathrm{cr}}$. Recall that the latter is defined as the scale at which $\bar{\nu}_{k}^{2}$ becomes zero, $\bar{\nu}_{k_{\text {cr }}}^{2}=0$. Employing the shape function (35), we can even compute the right-hand side of Eq. (96) analytically. In the limit $\mu / k \gg 1$, it then simplifies to $\partial_{t} \bar{\nu}_{k}^{2}=2 \mu^{2} / \pi^{2}$. This implies that $k_{\mathrm{cr}} \sim \exp \left(-c / \mu^{2}\right)$ for $\mu / \Lambda \ll 1$ where the $\mu$ independent constant $c>0$ depends on the initial condition of the flow equation at $k=\Lambda$. In other words, we recover the expected BCS-type scaling behavior of the critical scale, see also Eq. (29).

From this analysis of the flow equations in the $U_{\mathrm{V}}(1)$ symmetric regime, we observe that $\bar{\nu}_{k}^{2}$ changes its sign, indicating the onset of the formation of a diquark condensate. For $k<k_{\mathrm{cr}}$, we then expand the effective action around the scale-dependent ground state $\bar{\Phi}_{0}, \Gamma_{k} \sim \bar{\lambda}_{k}\left(\left|\Phi_{\mathrm{cl}}\right|^{2}-\left|\bar{\Phi}_{0}\right|^{2}\right)^{2}$, where $\left|\Phi_{\mathrm{cl}}\right|^{2}:=\sum_{A}\left|\Phi_{\mathrm{cl}, A}\right|^{2}$ and, for convenience, $\bar{\Phi}_{0}$ is chosen to point into the 2-direction of color space without loss of generality. Using this parametrization of the effective action, the flow equations for the low-energy regime read

$$
\begin{aligned}
\partial_{t}\left|\bar{\Phi}_{0}\right|^{2}= & \frac{2}{\bar{\lambda}_{k}} \int_{p} \tilde{\partial}_{t}\left(\frac{1}{p_{0}^{2}+(\mu-|\vec{p}|)^{2}\left(1+r_{+}\right)^{2}+\left|\bar{\Phi}_{0}\right|^{2}}\right. \\
& \left.+\frac{1}{p_{0}^{2}+(\mu+|\vec{p}|)^{2}\left(1+r_{-}\right)^{2}+\left|\bar{\Phi}_{0}\right|^{2}}\right)
\end{aligned}
$$

and 


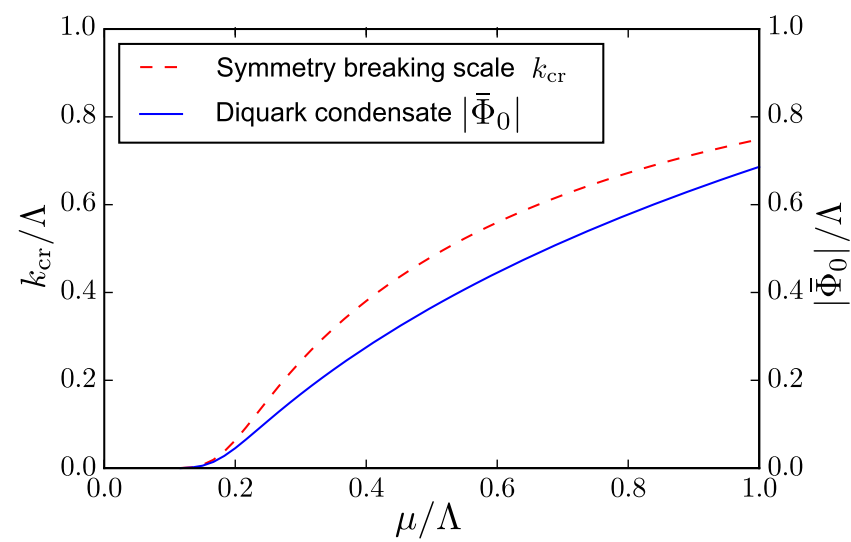

FIG. 3. Diquark gap $\left|\bar{\Phi}_{0}\right|$ and critical (symmetry breaking) scale $k_{\mathrm{cr}}$ in units of the UV scale $\Lambda$ as a function of the dimensionless chemical potential $\mu / \Lambda$.

$$
\begin{aligned}
\partial_{t} \bar{\lambda}_{k}= & 2 \int_{p} \tilde{\partial}_{t}\left(\left(\frac{1}{p_{0}^{2}+(\mu-|\vec{p}|)^{2}\left(1+r_{+}\right)^{2}+\left|\bar{\Phi}_{0}\right|^{2}}\right)^{2}\right. \\
& \left.+\left(\frac{1}{p_{0}^{2}+(\mu+|\vec{p}|)^{2}\left(1+r_{-}\right)^{2}+\left|\bar{\Phi}_{0}\right|^{2}}\right)^{2}\right) .
\end{aligned}
$$

We add that diquark self-couplings of higher order can be taken into account straightforwardly but this is beyond the scope of our present example study.

As an explicit example, we now compute the diquark gap of this model for $\left(\bar{\nu}_{\Lambda} / \bar{\nu}_{*}\right)^{2}=4 / 3$, where $\bar{\nu}_{*}^{2}$ is the value of $\bar{\nu}_{k}^{2}$ at the non-Gaußian fixed point, see also our detailed discussion in Sec. III. Phenomenologically speaking, this choice for $\bar{\nu}_{\Lambda}^{2}$ implies that the $U_{\mathrm{V}}(1)$ symmetry is only broken at finite $\mu$ but remains intact on all scales for $\mu=0$. In other words, the quarks are ungapped in the vacuum limit. For our numerical study of the flow equations, we employ the polynomial regulator function (62) with $N=4$ and set $\Lambda=0.6 \mathrm{GeV} .{ }^{10}$ We then find $\bar{\nu}_{*}^{2} / \Lambda^{2} \approx 0.065$.

Our numerical results for the critical scale $k_{\mathrm{cr}}$ and the value of the diquark gap $\left|\bar{\Phi}_{0}\right|$ for $k \rightarrow 0$ are shown in Fig. 3 . We observe that $k_{\mathrm{cr}}$ as well as the diquark gap $\left|\bar{\Phi}_{0}\right|$ exhibit the same qualitative behavior as a function of the chemical potential $\mu$. This may be viewed as a confirmation of the assumption $\left|\bar{\Phi}_{0}\right| \sim k_{\mathrm{cr}}$ from Sec. III, see Eq. (28). More specifically, for $\mu \rightarrow 0$, we observe that $\left|\bar{\Phi}_{0}\right|$ decreases exponentially whereas it increases monotonically for increasing chemical potential. These observations are in line with the expectation that $\bar{\Phi}_{0} \sim \exp \left(-c_{\bar{\phi}} / \mu^{2}\right)$ (with $c_{\bar{\phi}}>0$ ). For a detailed analysis of cutoff effects in this

\footnotetext{
${ }^{10}$ We have chosen $\Lambda=0.6 \mathrm{GeV}$ as it is a typically used value for the UV cutoff scale in low-energy model studies, see, e.g., Refs. $[16,87]$. Note also that our results shown in Fig. 3 remain qualitatively unchanged (BCS-type scaling of the diquark condensate and the critical scale) when we employ different regulator shape functions and values for the UV scale $\Lambda$, provided that we choose $\bar{\nu}_{\Lambda}^{2}$ to be greater than $\bar{\nu}_{*}^{2}$.
}

model, we refer the reader to Ref. [49]. As it is beyond the scope of the present work, we also do not present results for the pressure but only refer to Appendix A for a more general discussion of the computation of thermodynamic quantities.

Overall, we conclude that the class of regulators defined by Eq. (55) leads to well-behaved RG flows of the couplings in the presence of a Cooper instability, and the expected BCS-type scaling behavior of physical observables as a function of the chemical potential is recovered. Recall that the use of conventionally employed regulator functions leads to ill-defined RG flows, see Sec. III, which represented the starting point for the construction of the regulator functions employed in this section. Nevertheless, a word of caution regarding the regulator class defined by Eq. (55) is in order: this class is suited to study systems dominated by a Cooper instability in the long-range limit. In a regime which is governed by the Silver-Blaze property, an expansion of the effective action around a Silver-Blaze-symmetric point should be employed together with a regulator function that does not break the Silver-Blaze symmetry. For example, if we use regulators of the form proposed in Sec. IV to compute a diagram of the type as presented in Fig. 1 (right) with the external legs being now associated with scalar fields with vanishing fermion number $F$ (e.g., sigma or pion fields), then the diagram does not exhibit the correct scaling behavior as a function of the chemical potential. Note that, phenomenologically speaking, this scaling behavior is associated with the screening of interactions in, for example, the associated pseudoscalar channel when then chemical potential is increased. From this, it becomes apparent that the expansion point associated with a derivative expansion as well as the regularization scheme has to be chosen carefully depending on the external parameters, such as the chemical potential. In this sense, a derivative expansion with a given expansion point and regularization scheme should always be viewed as an effective description of a theory in terms of the most relevant degrees of freedom, and the expansion as well as the scheme should be chosen accordingly.

\section{CONCLUSIONS}

In the present work, we discussed issues arising in RG studies of dense systems, with a focus on the widely used derivative expansion of the effective action. Specifically, we critically assessed how to apply the derivative expansion to study dense-matter systems governed by a Cooper instability in a systematic fashion, including a discussion of the role played by the regularization scheme. In particular, we showed that a violation of the Silver-Blaze symmetry is required in a derivative expansion of the effective action in order to recover the correct BCS scaling behavior of physical observables. Based on these formal developments, we then introduced a new class of regulators which is 
suitable to tackle dense relativistic matter in the presence of a Cooper instability. This class of regulators agrees identically with the standard class of (three-dimensional) regulators in the vacuum limit. The latter property is convenient as model parameters are often fixed in the vacuum limit by fitting, for example, vacuum masses or scattering data. It is also worth mentioning that this new regulator class does not correspond to simply introducing a sharp cutoff around the Fermi surface. This is important as sharp cutoffs are known to lead to ambiguities in the evaluation of loop integrals beyond the lowest order of the derivative expansion. Finally, we demonstrated the application of this class of regulators with the aid of a quarkdiquark model and found the typical BCS-type exponential scaling behavior of the gap as function of the chemical potential.

While the use of our new class of regulators is certainly convenient for studies of matter in the presence of a Cooper instability, it should also be noted that it introduces an explicit breaking of the Silver-Blaze symmetry and is therefore not suited for studies of a regime at small chemical potential where the dynamics is strongly constrained by the Silver-Blaze property. This is not a specific problem of the constructed class of regulator functions but is, in general, the case when fluctuations are integrated out around the Fermi surface. In this sense, the constructed regulator class does not provide "multipurpose" regulators. Our present analysis rather suggests that a consistent and reliable description of the phase diagram and thermodynamics of strong-interaction matter based on a derivative expansion in the matter sector is, in general, not possible, if one insists on employing a derivative expansion anchored at the same point in momentum space for all densities. This can be traced back to the fact that a description of QCD requires to bridge the gap between a regime governed by chiral symmetry breaking at (very) low densities and the presence of a Cooper instability at intermediate and high densities. For baryon chemical potentials of the order of the mass of the nucleon or below, the dynamics is governed by the Silver-Blaze symmetry. Because of the latter, QCD should not exhibit at all a dependence on the baryon chemical potential as long as it remains smaller than the nucleon mass. In this regime (also at finite temperature), the derivative expansion should be anchored at a Silver-Blazesymmetric point in momentum space and, within the functional RG approach, regulators respecting the SilverBlaze symmetry should be employed. Note that it is not meaningful to expand the theory around the Fermi surface since the system does not depend on the chemical potential, at least for sufficiently small chemical potentials at zero temperature. At high densities, the situation is different. Here, the dynamics is expected to be governed by a Cooper instability. We showed that the derivative expansion should not be constructed around the Silver-Blaze-symmetric point, and also, fluctuations in the RG flow should be integrated out around the Fermi surface. The latter can be conveniently done with the new class of regulator functions introduced in the present work. Our discussion therefore suggests that it is required to switch the approximation scheme as a function of the chemical potential at some a priori unknown critical chemical potential. Above that point, by construction, we violate the Silver-Blaze symmetry in a description of dense strong-interaction matter based on a standard derivative expansion. Unfortunately, the regime around this critical chemical potential is most likely of great interest from a phenomenological standpoint as it probably "accommodates" the liquid-gas phase transition and the critical end point of the QCD phase diagram.

This may appear to be only a problem of RG approaches since the RG flow, in general, covers a wide range of scales, ranging from high momentum scales, $\mu / \Lambda \ll 1$, down to the low-momentum regime. With respect to other approaches, one may therefore be tempted to argue that the Silver-Blaze symmetry is broken anyhow in the infrared limit of QCD at large chemical potentials, as we argued above. Unfortunately, the issues discussed in the present work reach beyond RG studies. In fact, as we discussed, the violation of the Silver-Blaze symmetry also leaves its imprint in the counter terms in, for example, conventional loop expansions. Indeed, counter terms can only be chosen to be independent of the chemical potential, if the regularization scheme does not violate the Silver-Blaze symmetry.

We emphasize that we do not criticize the use of derivative expansions at all since studies resolving the full momentum dependence of correlation functions are very costly. We only would like to highlight issues potentially arising in a description of dense relativistic matter based on derivative expansions. At least some of them can be circumvented by carefully choosing the expansion point and scheme for a given density regime. With respect to QCD, our present analysis suggests that at least two approximation schemes (e.g., in terms of two derivative expansions anchored at two different points) are required to reliably describe the properties of strong-interaction matter over a wide range of densities and temperatures, without relying on a tuning of, for example, model parameters. Against this background, we believe that our present analysis of derivative expansions together with our new class of regulator functions for the functional $R G$ approach represent a step forward toward a quantitative description of dense strong-interaction matter.

\section{ACKNOWLEDGMENTS}

J. B. is grateful to Marc Leonhardt and Jan M. Pawlowski for many discussion on the subject of this work. As members of the fQCD Collaboration [88], J. B., B. S., and S. T. also would like to thank the other members of this collaboration for discussions. J. B. acknowledges support by the DFG under Grant No. BR 4005/4-1 and No. BR 4005/6-1 (Heisenberg program). J. B. and S. T. acknowledge support 
by BMBF under Grant No. 05P20RDFCA. This work is supported in part by the Deutsche Forschungsgemeinschaft (DFG, German Research Foundation)-Projektnummer 279384907-SFB 1245.

\section{APPENDIX A: THERMODYNAMICS}

In this Appendix, we would like to employ a noninteracting gas of single-component Dirac fermions to highlight peculiarities which arise in the computation of the pressure and are associated with the choice of the regularization scheme.

For a free relativistic Fermi gas at zero temperature, the path integral can be computed analytically,

$$
\Gamma_{0}=-\ln \mathcal{Z}_{\mathrm{gc}}=-2 V_{4 d} \int_{p} \ln \left(\left(p_{0}-\mathrm{i} \mu\right)^{2}+\vec{p}^{2}\right),
$$

where $V_{4 d}=\int \mathrm{d}^{4} x, \mathcal{Z}_{\mathrm{gc}}$ is the grand-canonical partition function, and $\Gamma_{0}$ is the effective action evaluated at its minimum. Note that the thermodynamic pressure $p_{T}$ is directly related to the effective action at its minimum $\Gamma_{0}$, $p_{T}=-\Gamma_{0} / V_{4 d}$. The integral in Eq. (A1) can be computed by suitably inserting an artificial parameter $m$ into the logarithm [89]

$$
\Gamma_{0}(m)=-2 V_{4 d} \int_{p} \ln \left(\left(p_{0}-\mathrm{i} \mu\right)^{2}+\vec{p}^{2}+m^{2}\right),
$$

where $m$ acts like a mass term for the fermions. We now take a derivative of this expression with respect to $m$,

$m \frac{\partial}{\partial m} \Gamma_{0}(m)=-4 m^{2} V_{4 d} \int_{p} \frac{1}{\left(p_{0}-\mathrm{i} \mu\right)^{2}+\vec{p}^{2}+m^{2}}$.

Note that it is unproblematic to take the first derivative with respect to $m$ as done here. However, higher-order derivatives may require special attention. In any case, after performing the integration with respect to $p_{0}$ and then with respect to $m$, we can set $m=0$ to perform the remaining integration with respect to the spatial momenta. We eventually obtain

$$
\frac{1}{V_{4 d}} \Gamma_{0}=-\frac{\mu^{4}}{12 \pi^{2}},
$$

where we dropped a divergent constant.

Let us now employ the Wetterich equation to compute the pressure of the free Fermi gas. The Wetterich equation for $\Gamma_{0}$ reads

$$
\partial_{t} \Gamma_{0, k}=-\operatorname{tr}_{\mathrm{D}} V_{4 d} \int_{p}\left(\partial_{t} R_{k}^{\psi}\right) \cdot\left(\Gamma_{\bar{\psi} \psi}^{(2)}+R_{k}^{\psi}\right)^{-1} .
$$

Here, the trace has to be taken with respect to the Dirac indices and

$$
\Gamma_{\vec{\psi} \psi}^{(2)}(p, q)=\left(-\left(p_{0}-\mathrm{i} \mu\right) \gamma_{0}-\vec{p}\right)(2 \pi)^{4} \delta^{(4)}(p-q) .
$$

In the present case, we can directly integrate the flow equation for $\Gamma_{0}$ since the two-point function does not depend on the RG scale $k$. Doing so, we arrive at

$$
\Gamma_{0}-\Gamma_{0, \Lambda}=-\operatorname{tr}_{\mathrm{D}} \int_{p}\left(\ln \Gamma_{\bar{\psi} \psi}^{(2)}-\ln \left(\Gamma_{\bar{\psi} \psi}^{(2)}+R_{\Lambda}^{\psi}\right)\right),
$$

where $\Gamma_{0} \equiv \Gamma_{0, k=0}$. It is worth adding that, even for a scaledependent two-point function, the integrated flow equation for $\Gamma$ can be written in a form similar to Eq. (A7), see Refs. [90,91] for a discussion. In any case, the first term on the right-hand side of Eq. (A7) contains the contribution $\sim \mu^{4}$ to the pressure. This becomes evident from a comparison with the right-hand side of Eq. (A1). The second term contains terms depending on the cutoff scale $\Lambda$ which are divergent in the limit $\Lambda \rightarrow \infty$.

For an explicit calculation of $\Gamma_{0}$, we have to specify the regulator function $R_{k}^{\psi /}$. Let us begin our computation of the pressure by employing the widely used class of conventional three-dimensional regulators defined in Eq. (32) which do not violate the Silver-Blaze symmetry. Employing the shape function (35) in Eq. (A5), we find

$k \frac{\partial}{\partial k} \Gamma_{0, k}=-4 k^{2} V_{4 d} \int_{p} \theta\left(k^{2}-\vec{p}^{2}\right) \frac{1}{\left(p_{0}-\mathrm{i} \mu\right)^{2}+k^{2}}$.

Recall that $\partial_{t}=k \partial_{k}$. From a comparison of Eq. (A8) with Eq. (A3), we deduce that $k$ has to some extent the effect of a mass gap in case of the conventional class of threedimensional regulators (32). Note that this is also true for the four-dimensional generalization of this regulator class [77-79]. In any case, the flow equation for $\Gamma_{0}$ can be solved analytically. We obtain

$$
\frac{1}{V_{4 d}} \Gamma_{0}=\frac{1}{V_{4 d}} \Gamma_{0, \Lambda}-\frac{\mu^{4}}{12 \pi^{2}}+\frac{\Lambda^{4}}{12 \pi^{2}},
$$

which agrees with Eq. (A4) up to an irrelevant constant. The latter is canceled by $\Gamma_{0, \Lambda}$. Thus, we recover the pressure $p_{T}=-\Gamma_{0} / V_{4 d}$ of the free relativistic Fermi gas.

It is worthwhile to add that also sharp cutoffs can be implemented in the functional RG approach. Following the discussion in Ref. [49], we can obtain a flow equation for $\Gamma_{0}$ for the (three-dimensional) sharp cutoff,

$k \frac{\partial}{\partial k} \Gamma_{0, k}=\frac{k^{3}}{\pi^{2}} V_{4 d} \int_{-\infty}^{\infty} \frac{\mathrm{d} p_{0}}{2 \pi} \ln \left(\left(p_{0}-\mathrm{i} \mu\right)^{2}+k^{2}\right)$.

From this, the thermodynamic pressure can be obtained by integrating over $k$ from $k=\infty$ to $k=0$, relabeling $k$ in $|\vec{p}|$, and then following the line of arguments which led us from Eq. (A1)-(A4). Note that a derivation of Eq. (A10) from Eq. (A5) is delicate as it requires the specification of a corresponding shape function for the regulator class defined in Eq. (32). For example, one may choose [64] 


$$
r_{\text {sharp }}=\frac{1}{\sqrt{\theta\left(\vec{p}^{2}-k^{2}\right)}}-1
$$

Other definitions also exist, see, e.g., Ref. [65]. Although the sharp regulator may be appealing at first glance since it allows to map functional RG flows onto conventional Wilson RG flows at one-loop order, it is clear that it should only be used with great care. In fact, already from the ambiguity in the definition of the shape function, it appears inevitable that ambiguities arise when this regulator is employed, see, e.g., Ref. [65] for a concrete example. These ambiguities then also leave their imprint in the predictions for the pressure, for example,in the prefactor of the term $\sim \mu^{4}$.

Let us now discuss the flow equation for $\Gamma_{0}$ as obtained when our new class of regulators defined by Eq. (55) is employed. For illustration purposes, we employ the shape function (35) which ensures that we recover the conventional three-dimensional regulators defined in Eq. (32). This simplifies the comparison with our considerations above, in particular, with Eq. (A8). The flow equation for $\Gamma_{0}$ then reads

$$
\begin{aligned}
k \frac{\partial}{\partial k} \Gamma_{0, k}= & -2 \mathrm{i} k V_{4 d} \int_{p}\left\{\theta\left(k^{2}-(|\vec{p}|+\mu)^{2}\right) \frac{1}{-p_{0}+\mathrm{i} k}\right. \\
& \left.+\theta\left(k^{2}-(|\vec{p}|-\mu)^{2}\right) \frac{\operatorname{sgn}(\mu-|\vec{p}|)}{-p_{0}+\mathrm{i} \operatorname{sgn}(\mu-|\vec{p}|) k}\right\} .
\end{aligned}
$$

First of all, we note that we recover the flow equation (A8) for $\mu=0$. For finite $\mu$, the two RG flows then differ by construction. In fact, the scale $k$ has no longer the effect of a mass gap but rather of a BCS-type gap. Moreover, the cutoff scales for particle and antiparticle excitations are now different and, as discussed in Sec. IV, the regulator class defined in Eq. (55) breaks explicitly the Silver-Blaze symmetry. Integrating the flow equation (A12), we find

$$
\frac{1}{V_{4 d}} \Gamma_{0}=\frac{1}{V_{4 d}} \Gamma_{0, \Lambda}+\frac{\Lambda^{4}}{12 \pi^{2}}+\frac{\Lambda^{2} \mu^{2}}{2 \pi^{2}} .
$$

The tree-level term $\Gamma_{0, \Lambda}$ can be computed. For the presently employed "Fermi-surface adapted" regulator (55) with the shape function (35), it reads

$$
\frac{1}{V_{4 d}} \Gamma_{0, \Lambda}=-\frac{\mu^{4}}{12 \pi^{2}}-\frac{\Lambda^{4}}{12 \pi^{2}}-\frac{\Lambda^{2} \mu^{2}}{2 \pi^{2}}+\ldots
$$

where we dropped terms vanishing in the limit $\Lambda \rightarrow \infty$. Thus, we also obtain the correct result for the pressure $p_{T}=-\Gamma_{0} / V_{4 d}$ from Eq. (A13).

Note that the presence of terms depending on $\Lambda$ and $\mu$ in Eq. (A13) is a generic feature of regulators which integrate out fluctuations around the Fermi surface [70].
Since the regulator class (55) effectively introduces a gap at the Fermi surface, we close by noting that the SilverBlaze symmetry is similarly broken in conventional meanfield studies of, for example, quark-diquark models. There, the direct inclusion of a background field with the quantum numbers of the gap in the calculation leads to a breaking of this symmetry [49].

\section{APPENDIX B: DERIVATION OF FLOW EQUATIONS FOR THE TWO-FLAVOR DIQUARK MODEL}

In this Appendix, we discuss the derivation of the RG flow equations presented in Eqs. (96)-(99) in Sec. V. To derive this set of flow equations, we have employed the following Ansatz for the scale-dependent quantum effective action $\Gamma_{k}$ :

$$
\begin{aligned}
\Gamma_{k}= & \int \mathrm{d}^{4} x\left\{\bar{\Psi}_{\mathrm{cl}}\left(\mathrm{i} \partial+\mathrm{i} \mu \gamma_{0}\right) \Psi_{\mathrm{cl}}+U_{k}\left(\Phi_{\mathrm{cl}, A}^{*} \Phi_{\mathrm{cl}, A}\right)\right. \\
& +\mathrm{i} \bar{\Psi}_{\mathrm{cl}} \gamma_{5} \tau_{2} \Phi_{\mathrm{cl}, A}^{*} T^{A} \mathcal{C} \bar{\Psi}_{\mathrm{cl}}^{T} \\
& \left.-\mathrm{i} \Psi_{\mathrm{cl}}^{T} \mathcal{C} \gamma_{5} \tau_{2} \Phi_{\mathrm{cl}, A} T^{A} \Psi_{\mathrm{cl}}\right\},
\end{aligned}
$$

where we choose

$$
U_{k=\Lambda}\left(\Phi_{\mathrm{cl}, A}^{*} \Phi_{\mathrm{cl}, A}\right)=\bar{\nu}_{k=\Lambda}^{2} \Phi_{\mathrm{cl}, A}^{*} \Phi_{\mathrm{cl}, A}
$$

as the initial condition at the scale $k=\Lambda$. This choice ensures that the initial condition $\Gamma_{k=\Lambda}$ for the Wetterich equation (i.e., the effective action at the initial scale) agrees with the classical action (27) evaluated on the classical fields. Recall that the implicit sum over the color index $A$ in, for example, Eq. (B1) runs only over the antisymmetric color generators $T^{A}$ (i.e., $A=2,5,7$ ) in the fundamental representation of $\mathrm{SU}(3)$.

In our illustrational study in Sec. $\mathrm{V}$, we only take into account the running of the effective potential as driven by loops with only fermionic internal lines. In particular, we drop a possible scale dependence of the Yukawa-type interaction term and the wave function renormalization factors. For a discussion of the running of the latter quantities, we refer the reader to Ref. [92]. Moreover, we only take into account up to quartic diquark selfinteraction terms in our Ansatz for the potential $U_{k}$. To be more specific, we employ

$$
U_{k}=\bar{\nu}_{k}^{2} \Phi_{\mathrm{cl}, A}^{*} \Phi_{\mathrm{cl}, A}+\bar{\lambda}_{k}\left(\Phi_{\mathrm{cl}, A}^{*} \Phi_{\mathrm{cl}, A}\right)^{2}
$$

for $k \geq k_{\mathrm{cr}}$ (i.e., in the symmetric regime) and

$$
U_{k}=\bar{\lambda}_{k}\left(\Phi_{\mathrm{cl}, A}^{*} \Phi_{\mathrm{cl}, A}-\left|\bar{\Phi}_{0}\right|^{2}\right)^{2}
$$

for $k<k_{\mathrm{cr}}$ (i.e., in the low-energy regime associated with the emergence of a finite diquark condensate $\bar{\Phi}_{0}$ ). For 
convenience, we choose $\Phi_{\mathrm{cl}, A, 0}=\left|\bar{\Phi}_{0}\right| \delta_{A, 2}$ where $\bar{\Phi}_{0}$ is assumed to be homogeneous and real valued.

In order to obtain flow equations for the quantities defined in our Ansätze for the potential $U_{k}$, we expand the Wetterich equation as follows:

$$
\begin{aligned}
\partial_{t} \Gamma_{k}= & \frac{1}{2} \operatorname{STr}\left(\partial_{t} R_{k}^{\psi}\right) \cdot\left(\Gamma_{\bar{\psi} \psi}^{(2)}+R_{k}^{\psi /}\right)^{-1} \\
= & \frac{1}{2} \operatorname{STr} \tilde{\partial}_{t} \ln \mathcal{P}_{k} \\
& -\frac{1}{2} \operatorname{STr} \tilde{\partial}_{t} \sum_{n=1}^{\infty} \frac{1}{n}\left(-\mathcal{P}_{k}^{-1} \mathcal{F}_{k}\right)^{n} .
\end{aligned}
$$

Here, $\Gamma_{\bar{\psi} \mu}^{(2)}$ is the second-derivative matrix associated with derivatives with respect to the fermion fields. The operator $\tilde{\partial}_{t}$ acts only on the $k$ dependence of the regulator. In this expansion, the regulator is fully absorbed in the inverse propagator $\mathcal{P}_{k}$ which is assumed to be evaluated on the ground state. Thus, in the low-energy regime, $\mathcal{P}_{k}$, in general, depends on $\bar{\Phi}_{0}$ and also $\bar{\lambda}_{k}$. The quantity $\mathcal{F}_{k}$ contains the fluctuations around the ground state. More specifically, we have used the following decomposition on the right-hand side of the Wetterich equation: $\Gamma_{\bar{\psi} \psi}^{(2)}+R_{k}^{\psi}=\mathcal{P}_{k}+\mathcal{F}_{k}$. For more details and specific examples, we refer the reader to Refs. [77,93] and, for an introduction, to Ref. [68].

Identifying now the complex fields $\Phi_{\mathrm{cl}, A}$ appearing in the ansatz (B3) for the potential with homogeneous background fields $\bar{\Phi}_{A}, \Phi_{\mathrm{cl}, A}=\bar{\Phi}_{A}$, we can extract the flow equation (96) for $\bar{\nu}_{k}^{2}$ from the expansion (B5) in the symmetric regime as follows:

$$
\partial_{t} \bar{\nu}_{k}^{2}=-\frac{1}{4 V_{4 d}}\left(\frac{\partial}{\partial\left(\bar{\Phi}_{2}^{*} \bar{\Phi}_{2}\right)} \operatorname{STr} \tilde{\partial}_{t}\left(\mathcal{P}_{k}^{-1} \mathcal{F}_{k}\right)^{2}\right)_{\mathrm{gs}} .
$$

Here, $V_{4 d}=\int \mathrm{d}^{4} x$ and the subscript "gs" (ground state) indicates that the right-hand side is evaluated on $\bar{\Phi}_{A}=0$. Note that, equivalently, we could take a derivative with respect to either $\left(\bar{\Phi}_{5}^{*} \bar{\Phi}_{5}\right)$ or $\left(\bar{\Phi}_{7}^{*} \bar{\Phi}_{7}\right)$ to obtain the flow equation for the quantity $\bar{\nu}_{k}^{2}$.

In the same way, we can also compute the flow equation for the four-diquark interaction in the symmetric regime,

$$
\partial_{t} \bar{\lambda}_{k}=-\frac{1}{16 V_{4 d}}\left(\frac{\partial^{2}}{\partial\left(\bar{\Phi}_{2}^{*} \bar{\Phi}_{2}\right)^{2}} \operatorname{STr} \tilde{\partial}_{t}\left(\mathcal{P}_{k}^{-1} \mathcal{F}_{k}\right)^{4}\right)_{\mathrm{gs}} .
$$

Again, other equivalent projections on the RG flow of the four-diquark coupling exist.

In the low-energy regime, the flow equations for the diquark condensate $\bar{\Phi}_{0}$ and the four-diquark coupling can be computed similarly. However, since we choose the ground state to point into the 2-direction, it turns out to be convenient to employ $\Phi_{\mathrm{cl}, A}=\bar{\Phi}_{A}=\delta \bar{\Phi}_{A}+\left|\bar{\Phi}_{0}\right| \delta_{A, 2}$ as a decomposition of the homogeneous background fields. The flow equation for the scale-dependent minimum $\left|\bar{\Phi}_{0}\right|^{2}$ of the effective action can then be obtained as follows:

$\partial_{t}\left|\bar{\Phi}_{0}\right|^{2}=\frac{1}{8 \bar{\lambda}_{k} V_{4 d}}\left(\frac{\partial}{\partial\left(\delta \bar{\Phi}_{5}^{*} \delta \bar{\Phi}_{5}\right)} \operatorname{STr} \tilde{\partial}_{t}\left(\mathcal{P}_{k}^{-1} \mathcal{F}_{k}\right)^{2}\right)_{\mathrm{gs}}$.

Here, the difference in the prefactors compared to the flow equation for $\bar{\nu}_{k}^{2}$ in Eq. (B6) can be traced back to the fact that we obtain $U_{k}=\ldots-2 \bar{\lambda}_{k} \delta \bar{\Phi}_{A}^{*} \delta \bar{\Phi}_{A}\left|\bar{\Phi}_{0}\right|^{2}+\cdots$, when we plug our decomposition of $\Phi_{A, \mathrm{cl}}$ into our ansatz for the potential for $k<k_{\mathrm{cr}}$. The subscript "gs" in Eq. (B8) now indicates that the right-hand side is evaluated on $\delta \bar{\Phi}_{A}=0$. Note that our choice for the decomposition of the homogeneous background fields $\bar{\Phi}_{A}$ also entails that it is required to take a derivative with respect to either $\left(\delta \bar{\Phi}_{5}^{*} \delta \bar{\Phi}_{5}\right)$ or $\left(\delta \bar{\Phi}_{7}^{*} \delta \bar{\Phi}_{7}\right)$ to project on the flow of $\left|\bar{\Phi}_{0}\right|^{2}$.

For the flow equation of the four-diquark interaction in the low-energy regime, we have

$\partial_{t} \bar{\lambda}_{k}=-\frac{1}{16 V_{4 d}}\left(\frac{\partial^{2}}{\partial\left(\delta \bar{\Phi}_{5}^{*} \delta \bar{\Phi}_{5}\right)^{2}} \operatorname{STr} \tilde{\partial}_{t}\left(\mathcal{P}_{k}^{-1} \mathcal{F}_{k}\right)^{4}\right)_{\mathrm{gs}}$.

This completes our discussion of the derivation of the flow equations (96)-(99) presented in Sec. V.

Finally, we would like to add that the expansion (B5) is convenient to also compute the flows of, for example, wave function renormalization factors and the running of Yukawa-type couplings. Within our present approximations, however, it is worth noting that the flow equation for the potential $U_{k}$ introduced in Eq. (B1) can be computed in closed form, i.e., without relying on Ansätze as given in Eqs. (B3) and (B4), respectively. To this end, we directly evaluate the Wetterich equation [first line of Eq. (B5)] on homogeneous complex-valued background fields $\bar{\Phi}_{A}$. In doing so, we eventually find

$$
\begin{aligned}
\partial_{t} U_{k}= & -4 \int_{p} \tilde{\partial}_{t}\left\{\ln \left(p_{0}^{2}+(\mu-|\vec{p}|)^{2}\left(1+r_{+}\right)^{2}+|\bar{\Phi}|^{2}\right)\right. \\
& \left.+\ln \left(p_{0}^{2}+(\mu+|\vec{p}|)^{2}\left(1+r_{-}\right)^{2}+|\bar{\Phi}|^{2}\right)\right\} .
\end{aligned}
$$

Here, $|\bar{\Phi}|^{2}=\sum_{A}\left|\bar{\Phi}_{A}\right|^{2}$, and we have dropped terms independent of the fields $\bar{\Phi}_{A}$ on the right-hand side.

The integration over the RG scale $k$ in Eq. (B10) can, in principle, be performed analytically since the only dependence of the right-hand side on $k$ originates from the regulator shape functions $r_{ \pm}$. Therefore, we have $\tilde{\partial}_{t} \equiv \partial_{t}=$ $k \partial_{k}$ and the right-hand side eventually becomes a total $k$ derivative. Note that it is, in general, not possible to solve the RG equation for the potential $U_{k}$ in closed form when bosonic fluctuation effects are taken into account. For such studies, recently developed techniques for the numerical 
solution of functional RG equations may turn out to be beneficial in the future [94-99].

In any case, the flow equation (B10) already describes diquark self-interactions of arbitrarily high orders as can be seen by an expansion of this equation in powers of $|\bar{\Phi}|^{2}$. In particular, it is instructive to see that we can recover the flow equations (96)-(99) from Eq. (B10). For example, the flow equations for the symmetric regime can be obtained by mapping Eq. (B10) onto the Ansatz (B3). For $\bar{\nu}_{k}^{2}$, it then follows that

$$
\partial_{t} \bar{\nu}_{k}^{2}=\left(\frac{\partial}{\partial|\bar{\Phi}|^{2}} \partial_{t} U_{k}\right)_{\bar{\Phi}=0} .
$$

This leads us to Eq. (96) as also Eq. (B6) does. In the same way, we can also derive the flow equation for the fourdiquark coupling $\bar{\lambda}_{k}$.
In the low-energy regime, where interactions generate a nontrivial ground state expectation value $\bar{\Phi}_{0} \neq 0$, we have to map the flow equation (B10) onto the Ansatz (B4) in order to recover the flow equations for $\left|\bar{\Phi}_{0}\right|^{2}$ and the fourdiquark coupling $\bar{\lambda}_{k}$. For example, using

$$
\partial_{t}\left|\bar{\Phi}_{0}\right|^{2}=-\frac{1}{2 \bar{\lambda}_{k}}\left(\frac{\partial}{\partial|\bar{\Phi}|^{2}} \partial_{t} U_{k}\right)_{\bar{\Phi}=\bar{\Phi}_{0}}
$$

we find the flow equation for $\left|\bar{\Phi}_{0}\right|^{2}$ presented in Eq. (98). Again, flow equations for couplings associated with higherorder diquark self-interaction channels can be obtained by taking a suitable number of derivatives of the flow equation (B10) with respect to $|\bar{\Phi}|^{2}$ and evaluating the resulting expression on $|\bar{\Phi}|^{2}=\left|\bar{\Phi}_{0}\right|^{2}$.
[1] D. Bailin and A. Love, Phys. Rep. 107, 325 (1984).

[2] M. G. Alford, K. Rajagopal, and F. Wilczek, Phys. Lett. B 422, 247 (1998).

[3] R. Rapp, T. Schäfer, E. V. Shuryak, and M. Velkovsky, Phys. Rev. Lett. 81, 53 (1998).

[4] D. T. Son, Phys. Rev. D 59, 094019 (1999).

[5] T. Schäfer and F. Wilczek, Phys. Lett. B 450, 325 (1999).

[6] J. Berges and K. Rajagopal, Nucl. Phys. B538, 215 (1999).

[7] T. Schäfer and F. Wilczek, Phys. Rev. D 60, 114033 (1999).

[8] R. D. Pisarski and D. H. Rischke, Phys. Rev. D 61, 074017 (2000).

[9] R. D. Pisarski and D. H. Rischke, Phys. Rev. D 61, 051501 (2000).

[10] W. E. Brown, J. T. Liu, and H.-c. Ren, Phys. Rev. D 61, 114012 (2000).

[11] D. K. Hong, V. A. Miransky, I. A. Shovkovy, and L. C. R. Wijewardhana, Phys. Rev. D 61, 056001 (2000); 62, 059903 (E) (2000).

[12] S. D. Hsu and M. Schwetz, Nucl. Phys. B572, 211 (2000).

[13] N. J. Evans, J. Hormuzdiar, S. D. H. Hsu, and M. Schwetz, Nucl. Phys. B581, 391 (2000).

[14] K. Rajagopal and F. Wilczek, The condensed matter physics of QCD, in At the Frontier of Particle Physics, edited by M. Shifman and B. Ioffe, Handbook of QCD Vol. 1-3, (World Scientific, 2000), pp. 2061-2151.

[15] M. G. Alford, Annu. Rev. Nucl. Part. Sci. 51, 131 (2001).

[16] M. Buballa, Phys. Rep. 407, 205 (2005).

[17] I. A. Shovkovy, Found. Phys. 35, 1309 (2005).

[18] M. G. Alford, A. Schmitt, K. Rajagopal, and T. Schäfer, Rev. Mod. Phys. 80, 1455 (2008).

[19] K. Fukushima and T. Hatsuda, Rep. Prog. Phys. 74, 014001 (2011).

[20] K. Fukushima, J. Phys. G 39, 013101 (2012).
[21] R. Anglani, R. Casalbuoni, M. Ciminale, N. Ippolito, R. Gatto, M. Mannarelli, and M. Ruggieri, Rev. Mod. Phys. 86, 509 (2014).

[22] A. Schmitt, Lect. Notes Phys. 888, 1 (2015).

[23] J. Braun, M. Leonhardt, and M. Pospiech, Phys. Rev. D 97, 076010 (2018).

[24] J. Braun, M. Leonhardt, and M. Pospiech, Phys. Rev. D 101, 036004 (2020).

[25] R. Shankar, Rev. Mod. Phys. 66, 129 (1994).

[26] M. Salmhofer, C. Honerkamp, W. Metzner, and L. Oliver, Prog. Theor. Phys. 112, 943 (2004).

[27] S. Diehl, S. Floerchinger, H. Gies, J. M. Pawlowski, and C. Wetterich, Ann. Phys. (Berlin) 522, 615 (2010).

[28] W. Metzner, M. Salmhofer, C. Honerkamp, V. Meden, and K. Schonhammer, Rev. Mod. Phys. 84, 299 (2012).

[29] J. Braun, L. M. Haas, F. Marhauser, and J. M. Pawlowski, Phys. Rev. Lett. 106, 022002 (2011).

[30] N. Strodthoff, B.-J. Schaefer, and L. von Smekal, Phys. Rev. D 85, 074007 (2012).

[31] N. Strodthoff and L. von Smekal, Phys. Lett. B 731, 350 (2014).

[32] K. Morita, B. Friman, K. Redlich, and V. Skokov, Phys. Rev. C 88, 034903 (2013).

[33] K.-I. Aoki, S.-I. Kumamoto, and D. Sato, Prog. Theor. Exp. Phys. 2014, 043 B05 (2014).

[34] N. Khan, J. M. Pawlowski, F. Rennecke, and M. M. Scherer, arXiv:1512.03673.

[35] C. Jung, F. Rennecke, R.-A. Tripolt, L. von Smekal, and J. Wambach, Phys. Rev. D 95, 036020 (2017).

[36] T. Yokota, T. Kunihiro, and K. Morita, Prog. Theor. Exp. Phys. 2016, 073D01 (2016).

[37] W.-j. Fu, J. M. Pawlowski, F. Rennecke, and B.-J. Schaefer, Phys. Rev. D 94, 116020 (2016).

[38] F. Rennecke and B.-J. Schaefer, Phys. Rev. D 96, 016009 (2017). 
[39] G. A. Almasi, B. Friman, and K. Redlich, Phys. Rev. D 96, 014027 (2017).

[40] W.-j. Fu, J. M. Pawlowski, and F. Rennecke, Phys. Rev. D 100, 111501 (2019).

[41] W.-j. Fu, J. M. Pawlowski, and F. Rennecke, Phys. Rev. D 101, 054032 (2020).

[42] K. Otto, M. Oertel, and B.-J. Schaefer, Phys. Rev. D 101, 103021 (2020).

[43] R.-A. Tripolt, D. H. Rischke, L. von Smekal, and J. Wambach, Phys. Rev. D 101, 094010 (2020).

[44] J. Braun, W.-j. Fu, J. M. Pawlowski, F. Rennecke, D. Rosenblüh, and S. Yin, Phys. Rev. D 102, 056010 (2020).

[45] N. Dupuis, L. Canet, A. Eichhorn, W. Metzner, J. Pawlowski, M. Tissier, and N. Wschebor, Phys. Rep. 910, 1 (2021).

[46] M. Leonhardt, M. Pospiech, B. Schallmo, J. Braun, C. Drischler, K. Hebeler, and A. Schwenk, Phys. Rev. Lett. 125, 142502 (2020).

[47] G. Markó, U. Reinosa, and Z. Szep, Phys. Rev. D 90, 125021 (2014).

[48] T. D. Cohen, Phys. Rev. Lett. 91, 222001 (2003).

[49] J. Braun, M. Leonhardt, and J. M. Pawlowski, SciPost Phys. 6, 056 (2019).

[50] W.-j. Fu and J. M. Pawlowski, Phys. Rev. D 92, 116006 (2015).

[51] C. Wetterich, Phys. Lett. B 301, 90 (1993).

[52] A. Roberge and N. Weiss, Nucl. Phys. B275, 734 (1986).

[53] S. Floerchinger, J. High Energy Phys. 05 (2012) 021.

[54] M. Haas, L. Fister, and J. M. Pawlowski, Phys. Rev. D 90, 091501 (2014).

[55] R.-A. Tripolt, N. Strodthoff, L. von Smekal, and J. Wambach, Phys. Rev. D 89, 034010 (2014).

[56] R.-A. Tripolt, L. von Smekal, and J. Wambach, Phys. Rev. D 90, 074031 (2014).

[57] J. M. Pawlowski and N. Strodthoff, Phys. Rev. D 92, 094009 (2015).

[58] J. M. Pawlowski, N. Strodthoff, and N. Wink, Phys. Rev. D 98, 074008 (2018).

[59] J. Braun, M. Leonhardt, and M. Pospiech, Phys. Rev. D 96, 076003 (2017).

[60] J. Braun, K. Schwenzer, and H.-J. Pirner, Phys. Rev. D 70, 085016 (2004).

[61] B.-J. Schaefer and J. Wambach, Nucl. Phys. A757, 479 (2005).

[62] J.-P. Blaizot, A. Ipp, R. Mendez-Galain, and N. Wschebor, Nucl. Phys. A784, 376 (2007).

[63] D. F. Litim and J. M. Pawlowski, J. High Energy Phys. 11 (2006) 026.

[64] J. M. Pawlowski, Ann. Phys. (Amsterdam) 322, 2831 (2007).

[65] J. Braun, H. Gies, L. Janssen, and D. Roscher, Phys. Rev. D 90, 036002 (2014).

[66] M. C. Birse, B. Krippa, J. A. McGovern, and N. R. Walet, Phys. Lett. B 605, 287 (2005).

[67] S. Friederich, H. C. Krahl, and C. Wetterich, Phys. Rev. B 83, 155125 (2011).

[68] J. Braun, J. Phys. G 39, 033001 (2012).
[69] D. Roscher, J. Braun, and J. E. Drut, Phys. Rev. A 91, 053611 (2015).

[70] J. M. Pawlowski, M. M. Scherer, R. Schmidt, and S. J. Wetzel, Ann. Phys. (Amsterdam) 384, 165 (2017).

[71] J. Braun, Phys. Rev. D 81, 016008 (2010).

[72] D.F. Litim and J.M. Pawlowski, On gauge invariant Wilsonian flows, in The Exact Renormalization Group, edited by A. Krasnitz, Y. A. Kubyshin, R. Potting, and P. Sá, (World Scientific, Singapore, 1999), pp. 168-185.

[73] H. Gies, Lect. Notes Phys. 852, 287 (2012).

[74] J. D. Bjorken and S.D. Drell, Relativistic Quantum Mechanics, International Series in Pure and Applied Physics (McGraw-Hill, New York, 1965).

[75] S. Pokorski, Gauge Field Theories (Cambridge University Press, United Kingdom, 1987).

[76] T. Schäfer, Nucl. Phys. A728, 251 (2003).

[77] D. U. Jungnickel and C. Wetterich, Phys. Rev. D 53, 5142 (1996).

[78] J. Berges, D. U. Jungnickel, and C. Wetterich, Phys. Rev. D 59, 034010 (1999).

[79] J. Berges, N. Tetradis, and C. Wetterich, Phys. Rep. 363, 223 (2002).

[80] D. F. Litim, Phys. Lett. B 486, 92 (2000).

[81] D. F. Litim, Int. J. Mod. Phys. A 16, 2081 (2001).

[82] D. F. Litim, Phys. Rev. D 64, 105007 (2001).

[83] D. F. Litim and J. M. Pawlowski, Phys. Lett. B 546, 279 (2002).

[84] H. Gies, Phys. Rev. D 66, 025006 (2002).

[85] H. Gies and J. Jaeckel, Phys. Rev. Lett. 93, 110405 (2004).

[86] J. Braun and H. Gies, J. High Energy Phys. 06 (2006) 024.

[87] S. P. Klevansky, Rev. Mod. Phys. 64, 649 (1992).

[88] J. Braun, Y.-r. Chen, W.-j. Fu, F. Ihssen, A. Geißel, J. Horak, C. Huang, J. M. Pawlowski, F. Rennecke, F. Sattler, B. Schallmo, C. Schneider, Y.-y. Tan, S. Töpfel, R. Wen, N. Wink, and S. Yin (fQCD Collaboration) (members as of October 2021).

[89] M. L. Bellac, Thermal Field Theory, Cambridge Monographs on Mathematical Physics (Cambridge University Press, Cambridge, England, 2011).

[90] J. Braun, H. Gies, and J. M. Pawlowski, Phys. Lett. B 684, 262 (2010).

[91] J. Braun, A. Eichhorn, H. Gies, and J. M. Pawlowski, Eur. Phys. J. C 70, 689 (2010).

[92] J. Braun and B. Schallmo, arXiv:2106.04198.

[93] H. Gies and C. Wetterich, Phys. Rev. D 65, 065001 (2002).

[94] E. Grossi and N. Wink, arXiv:1903.09503.

[95] E. Grossi, F. J. Ihssen, J. M. Pawlowski, and N. Wink, Phys. Rev. D 104, 016028 (2021).

[96] A. Koenigstein, M. J. Steil, N. Wink, E. Grossi, J. Braun, M. Buballa, and D. H. Rischke, arXiv:2108.02504.

[97] A. Koenigstein, M. J. Steil, N. Wink, E. Grossi, and J. Braun, arXiv:2108.10085.

[98] M. J. Steil and A. Koenigstein, arXiv:2108.04037.

[99] J. Stoll, N. Zorbach, A. Koenigstein, M. J. Steil, and S. Rechenberger, arXiv:2108.10616. 